\title{
New Properties of HM16 Ether, with Submicroparticles as Self-Functional Cells Interacting through Percussion Forces, Establishing Nature of Electrical Charges, including Gravitation
}

\author{
Ioan Has ${ }^{1}$, Simona Miclaus ${ }^{1}$, Aurelian Has ${ }^{2}$ \\ ${ }^{1}$ Land Forces Academy "Nicolae Balcescu”, Sibiu, Romania \\ ${ }^{2}$ Independent Researcher, Rm. Valcea, Romania \\ Email: hasavo@yahoo.com,simo.miclaus@gmail.com, hasaurelian@yahoo.com
}

How to cite this paper: Has, I., Miclaus, S. and Has, A. (2020) New Properties of HM16 Ether, with Submicroparticles as Self-Functional Cells Interacting through Percussion Forces, Establishing Nature of Electrical Charges, including Gravitation. Journal of Modern Physics, 11, 803-853.

https://doi.org/10.4236/jmp.2020.116052

Received: April 27, 2020

Accepted: May 31, 2020

Published: June 3, 2020

Copyright $\odot 2020$ by author(s) and Scientific Research Publishing Inc. This work is licensed under the Creative Commons Attribution International License (CC BY 4.0).

http://creativecommons.org/licenses/by/4.0/

\begin{abstract}
Article continues and complements our previous articles on the HM16 ether (ETH) model. Here, we describe the mechanism of occurrence of the submicroparticle (SMP). A general hypothesis, HFVI, is introduced for the modalities of interaction between two SMPs, based on periodic mechanical percussion forces, produced by fundamental vibrations FVs. A mechanism for describing the interaction between a SMPs and the ETH is presented. Positive and negative particles are defined by their membrane types of movement, such as,$+-u /+,-V$ vibrations, and rotations at speeds $+\Omega /-\Omega$. The process of creating a pair of SMPs is discussed. Applying HFVI to the interaction between pairs of SMPs immobile in ETH, and considering longitudinal FVL, was obtained the forces of attraction/repulsion $+F_{L 21} /-F_{L 21}$, which correspond to the completed Coulomb force $F_{C C}$ including gravitation. The resultant $F_{\mathrm{RL} 21}$ will form an oriented field of forces, which is a quasielectric field $Q E$, equivalent to actual $E$ electric field. Considering transversal FVT, was obtained the vibratory forces,$+-F_{T 21}$, whose resultant forms an vibrating field of forces, $Q H s$, a quasimagnetic special field, which may explain some of the quantum properties of SMPs. Considering a mobile SMP, two new $\gamma$ strains in ETH appear. Strains $\gamma_{L}$ are created by the displacement of SMP with velocity $V$, whose force,$+-F_{T 12}$ is the support of a component of the magnetic field $H$ (quasimagnetic field $Q H$ ), giving the $Q H_{L}$ component. Strains $\gamma_{\mathrm{R}}$ are created by the rotation of SMP with speed $\Omega$, whose force,$+-F_{\mathrm{R} 12}$ constitutes physical support of the component $Q H_{R}$ of magnetic field $H$ (i.e. $Q H$ ). The crea-
\end{abstract}


tion of a photon $\mathrm{PH}$ is modelled as a special ESMP containing two zones of opposed rotations, and a mechanism is presented for its movement in the ETH with speed $c$ based on the HS hypothesis of screwing in ETH, with frequency $v$.

\section{Keywords}

Nature of Electrical Charges, Submicroparticle Constitution, Microparticle Interaction by Percussions, Ether Model HM16 with Fundamental Vibrations, Completed Coulomb's Law, Photon Constitution and Travel

\section{Composition of Submicroparticles in the Form of Complex Self-Functional Cells}

The HM16 ether model, originally proposed by the present authors in 2016 [1], will be used as the starting point in this article for development regarding the composition, behaviour and effects of the ETH in nature, and particular in physics. We will use the abbreviations used in [1], and these will be redefined and completed here.

We use the following terminology: MP: microparticle, SMP: submicroparticle, SMPP: positive submicroparticle (rather than $\mathrm{SMP}^{+}$), SMPN: negative submicroparticle (rather than $\mathrm{SMP}^{-}$), ESMP: elementary or special submicroparticle, PH: photon, RF: reference frame, EC: base cells of the ether, PC: constituent cell of an SMP, EP: ether constituents (etherons $\alpha, \beta$ ), and MB: material body.

We will also refer to neutral SMPs as SMPNEs. These include neutrons, and consist of SMPP and SMPN types, in pairs (Figure 1).

We denote the ether as ETH, with its customisation, the HM16 model, which contains the fundamental vibrations FV of the EC cells of free ETH. PV is used to denote the vibrations of PC cells inside any type of microparticle (MP). $F_{C C}$ indicates the corrected/completed Coulomb electrical force, while $F_{C}$ represents the classical electrical Coulomb force; $F_{N}$ means Newton's classical gravitation force, and $F_{\mathrm{DC}}$ denotes the corrected electrical force between two electrical dipoles that actually constitute gravity [2] [3]. We use $\varepsilon$ and $\gamma$ to represent the specific/unitary linear and angular/tangential deformations (strains), while $\sigma$ and $\tau$ represent the normal and tangential unitary forces (stresses), respectively.

It should be noted that in HM16, all types of MPs, including SMPs and ESMPs, have the composition of an SMP autonomous cell (SMPAC) at the internal organisational level, as they are complex and self-functional in a similar way to live organism cells (LOCs).

Although we present SMPs as having a simple spherical shape, they may also take on more complex forms such as cylinders, ellipsoids, stars, neurons, etc., since SMPs form the most complex existing body, the universe, which requires interconnectable foundation bricks of similar complexity (Figure 1). 


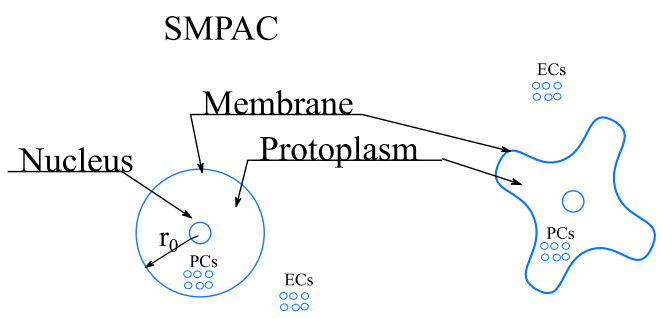

a).
SMPNE/NEUTRON

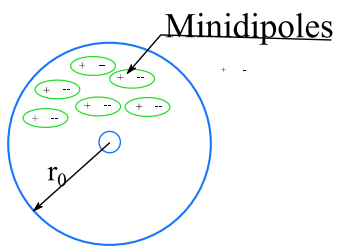

b).

Figure 1. SMPAC shape model of SMPs. (a) SMPS spherical and neuronal types; (b) Neutron type.

This composition of the particles of all known existing matter mainly involves the make-up of all SMPs in nature, in the form of SMPACs, and we call this the hypothesis of cellular organisation (HCO) of SMPs.

In the HCO, SMPAC cells have well-known LOC substructures, including a nucleus (NC), protoplasm (PP), and a membrane (MM) (Figure 1). The MM is primarily responsible for protecting the integrity of the SMP and preserving its number of substructures, all having an ethereal nature. The role of the MM is to preserve its own energy $E$, and thus to ensure the functional maintenance of the SMP. The MM also determines the possible interaction, contact and association of SMP with other surrounding SMPs or MPs, and ensures permanent contact with the first row of ECs from free ETH, or even with basic etherons (ETs), so constituting the $S$ surface of SMP, noted $S / M M$.

The MM is also responsible for facilitating exchanges of the etheronic material of ECs, energy ( $E$ ) or information (INF) (related to energy) between SMPs or between MPs.

In the HCO, the PP constitutes the basic matter of the SMP, and is made up of PCs. However, these PCs are formed of ETs, which make up the internal cells of the PC. PP ensures lossless storage of the energy $E_{p}$ of the SMP, and consists of permanent vibrations/vortices with rotation speed $\omega$, of the internal PCs ethereal cells. The PP therefore directly participates in any changes or exchanges of energy, and even of ECs cells with external ETH, or with other SMPs, thus ensuring the functionality of the SMP.

The PP also has the primary role of preserving the SMP's mass $m$ through the PCs it contains; this mass will need to be defined in the future.

In this model, the NC core is responsible for recording and storing the footprint (FP) of the SMP in terms of the nature of the information recorded internally in the NC. The NC stores and processes information exchanged with the ether and with the SMPs in the external environment. It also determines by commands the actions/reactions of the SMP and creates the reactions of the SMP to INF received from the outside, including specific reactions of attraction or rejection, as part of the process of interaction between the SMPP and SMPN (Sections 3, 4).

To achieve this, the NC core has a DNA composition in the same way as in an 
LC cell, due to the formation of the SMP, which allows it to perform these smart functions.

It follows from the HCO that MPs, including SMPs, have the same behaviour or manifestations as LC entities, and are in a permanent state of vibration and movement, and therefore should not be regarded as inert or "dead" particles.

\section{HFVI Hypothesis: Interaction between SMPs via Percussive Forces, Given by FVLs and FVTs}

In our original 2016 paper on the HM16 ether model [1], we discussed the ability of MPs and SMPs to transmit FVs to the ETH around them, thus justifying interactions between SMPs in principle. Today, these interactions are considered to be electrical or gravitational forces, although the actual physical nature of these two interactions and forces and the concrete mechanism of interaction via mechanical forces acting between SMPs have not yet been explained.

In this article, we present and discuss in detail a new complex hypothesis called HFVI, which involves the fundamental vibration interactions and the mechanism for interaction between SMPs via the percussive forces created by the FVs of the ETH, permanently acting in the ETH, and between all SMPs.

\subsection{General Framework for the Implementation of HFVI}

We first admit the mechanical interactions unity principle (MIUP), according to which an interaction force $F$ (here, $F, p, p_{s}, \sigma$, $\tau$, etc.) and the accompanying deformation $\delta$ (here, $\Delta x, u, v, u_{s}, v_{s} \mathcal{E}, \gamma$, etc.) are inseparable, as a moment $t_{i}$ in time, and as the point $M_{i}$ of the interaction in space (ether). The presence of one of these parts logically implies the presence of the other part.

In HFVI, these interactions between SMPs via FVs occur exclusively through mechanical actions of periodic percussion forces,$+-p_{p}$, which act discretely at the SMP level. We consider here that the percussion forces,$+-p_{p}$ are the only real actions that are physically possible, both in the case of interactions between MBs in nature and interactions between SMPs in physics, including electromagnetism, atomic physics and quantum physics. We assume that any force $F$ in physics or nature that is considered to be continuous, in fact is based on periodic percussion forces,$+-p_{p}$. These periodic percussion forces form the actual physical basis of quantum mechanical interactions.

It should be noted that this mechanism of percussion forces,$+-p_{p}$ that in HFVI explains the behaviour of SMPs and their interactions via FVs has not been confirmed on an experimental basis at the SMP scale; only laboratory-scale experiments have been carried out, and we rely on these here. This is the reason for the development in this paper of a physical mechanism of interaction at SMP scale through percussion forces,$+-p_{p}$, in the form of the HFVI hypothesis.

However, our HFVI can be initially confirmed by ensuring the functionality of the HM16 ether model based on the results of analyses of detailed interactions between SMPs, as discussed later in this article. 
It should be noted that HFVI is not consistent with currently accepted concepts in mainstream physics, as it does not allow for the existence of the ether and instead assumes the validity of the SRT and GR. However, as we have previously demonstrated theoretically [4] [5] [6] [7] [8], these two theories are beautiful mathematical inventions that correctly describe the behaviour of SMPs at the macro scale, but do not describe the interactions at SPMs scale in a mechanical sense. This is similar to the situation created by Ptolemy's geocentric theory, which for many centuries was able to explain the movements of the planets on a large scale via the geometric contrivance of epicycles, but which ultimately proved to be inconsistent with observable astronomical reality.

Our hypothesis (HFVI) for the action of FVs arises from the actual behaviour of continuous elastic crystalline bodies (ECBs), which we propose are similar to our model of the type A ether, called HM16 [1]. These ECBs are found in daily life on a human scale in many forms, for example in natural rocks, manufactured metals, man-made buildings, industrial materials, etc.

In our previous paper [1], we postulated that the ETH is also a real natural elastic body with a special nature or composition and somewhat ideal properties, which are as yet unknown from direct study. These properties are known only indirectly from observations of the interactions between ETH and ordinary matter (still noted with OM). It follows that the mechanical behaviours and properties of these two bodies, the ETH and the ECB, can and must be common and similar.

\subsection{Similarities between the Properties of Elastic Body ECB and the ETH}

It is known from mechanics that an ECB will undergo relative displacements of its component particles through deformations of its shape, if it is acted on at one of its points $M$ at some time $t$ by a local instantaneous force on a small area, called a percussion force or simply percussion, $p$.

We will assume that these percussions are always periodic forces $p=+,-p_{p}$, if they act for a finite period of time on the ECB.

As a general rule, we will consider that the sign of any force $F$, including the percussion,$+-p_{p}$, will be positive $(+/ \mathrm{P})$ if it produces tension on the surface on which it acts and vice versa, i.e. a force will be negative $(-/ \mathrm{N})$ if it causes compression at that surface.

It is known that the percussion forces acting on an ECB can be of two types: normal forces on the surface on which they act, denoted here as,$+-p_{n}$ (with the related unit force or stress,$+-\sigma$ ) and tangential forces at the surface, denoted as ,$+-p_{t}$ (with the related unit force or stress $\tau$ ). Depending on the significance of the force, we can also use other notations and indices.

The deformations in the ECB created by a periodic force,$+-p_{p}$ will have the general forms known from mechanics: linear deformations $u$, created by $p_{n}$ forces, and shear angular deformations $v$, created by $p_{t}$ forces. These $u$ and $v$ deformations will create in the ECB specific deformations or strains, that are linear 
$(\varepsilon)$ and angular $(\gamma)$, respectively, and these are also periodic.

These two types of deformations in the ECB environment (linear $u / \varepsilon$ and angular $v / \gamma$ ) may occur in any combination, i.e. separately ( $u / \varepsilon$ or $v / \gamma)$, or together. We assume in HFVI that in the special physical body of the ETH, a third combination is also possible over time, at any point in space.

A real situation in mechanics and nature that is similar to an SMP within the ETH is the spatial deformation of a body/environment caused by an explosion or shock/percussion at a point $M$ inside a massive ECB. In this context, we can mention the explosion caused by a bomb or shell in the air, water or earth, the explosion of dynamite in a mountain to create a tunnel, or the more well-known and well-studied case of the onset of an earthquake in the rock of the earth's crust.

The way in which waves are transmitted in an ECB environment is very well illustrated by seismic waves, which appear and are transmitted through the earth's crust in all directions in spatial/spherical form, starting from a point called the hypocentre at which the earthquake originated. In this case, $u$-type longitudinal waves are also called principal waves $(P)$, and the $v$-type transversal waves are called secondary waves $(S)$; these are known to spread independently and with different propagation speeds, $V_{\mathrm{P}}$ and $V_{\mathrm{S}}$, respectively, within the rock of the earth's crust [9].

Obviously, the similarity with the ECB/ETH system is only partial, since the two cases involve different phenomena; in the case of explosions or earthquakes, the short duration does not allow the static equilibrium steady state described in Section 2.3.2 (Figure 3) to be achieved, and energy transport will be present in the ECB. However, the deformations of types $u$ and $v$ are similar.

\subsection{Mode of Functioning of SMPs in the ETH in HFVI}

\subsubsection{SMP Behaviour Exposing Deformations $u_{s}$ and $v_{s}$ Creating FVs in the ETH \\ 1) Case of deformations $u_{s}$ :}

In HFVI, we postulate that the ETH is made up internally of individual ECs with bonds between them that are elastic at small deformations to form a continuous and infinite body, whose overall behaviour can be represented by the HM16 [1] model.

We consider the situation where an SMP1 is present at a point $M=\mathrm{O}_{1}$ in the ETH at time $t_{0}$, whose outer surface/membrane $S / M M$ we assume to be near spherical; the surface $S$ is represented by the cell membrane MM in HCO.

For the purpose of positioning the physical elements in space, any point $M_{i}$ in space is represented in HFVI using a Cartesian coordinate system Oxyz (Figure 2 ), with the origin fixed at a point $\mathrm{O}$ attached to an object/SMP, whose velocity $V$ relative to the ETH is considered to be known. The orientation of the $\mathrm{x}, \mathrm{y}, \mathrm{z}$ axes is chosen based on the directions of symmetry of the crystalline structure of the ether, which we assume are known.

We also assume in HFVI that the surface $S / M M$ exhibits normal/radial vibra- 
tions,$+-u_{s}$ with frequency $f_{n}$. As a result, we assume that $S / M M$ is the source of periodic normal deformations,$+-u$ in surrounding ETH.

But $S / M M$ also rotates around the $\mathrm{O} z$ axis with rotational speed $\Omega$ (frequency $f_{t}$ ) (Figure 2). As a result, we assume that $S / M M$ is the source of periodic tangential deformations,$+-v$ in surrounding ETH after the subsequent mechanism.

The vibrational deformations of SMP1, denoted by,$+-u_{s}$ (Figure 2 ), are considered to be normal at $S / M M$. They are therefore radial, with alternate expansion and contraction, and have the same spherical symmetry as the SMP1.

The,$+-u_{s}$ deformations at the $S / M M$ surface of SMP1 will act directly on the first layer $r_{1}$ of the ECs in the ETH that are in contact with $S / M M$ (Figure 2), producing equivalent deformations,$+-u$ in the ECs from the first row $r_{1}$ of the ETH cells. Obviously, at $S$, we have:

$$
+,-u=+,-u_{s}
$$

Due to the direct contact and links between all ECs in the ETH, deformations ,$+-u$ in row $r_{2}$ of the ECs will also occur, and then deformations in row 3 of the cells, and so on ad infinitum (Figure 2). These deformations,$+-u$ will be transmitted in the ETH from $S / M M$ to infinity, creating longitudinal fundamental vibrations FVL. We note that,$+-u$ are not influenced by the rotational speed $\Omega$ of SMP.

\section{2) Case of deformations $v_{s}$ :}

The rotations with speed $\Omega$ of the $S / M M$ surface of SMP1 are considered to be circular (i.e. continuously rotating) around an axis $\mathrm{O} z$, after the parallel circles of $S$, and produce in combination with,$+-u_{s}$, the equivalent/reactive tangential deformations in $S / M M$ denoted by,$+-V_{s}$ according to the mechanism described below (Figure 2).

Thus, at the contact points between $S / M M$ and the outer ETH in crystalline form, which in HM16 is assumed to be immobile, a series of periodic attachments and detachments will occur between $S / M M$, and the $1^{\text {st }}$ layer of crystalline

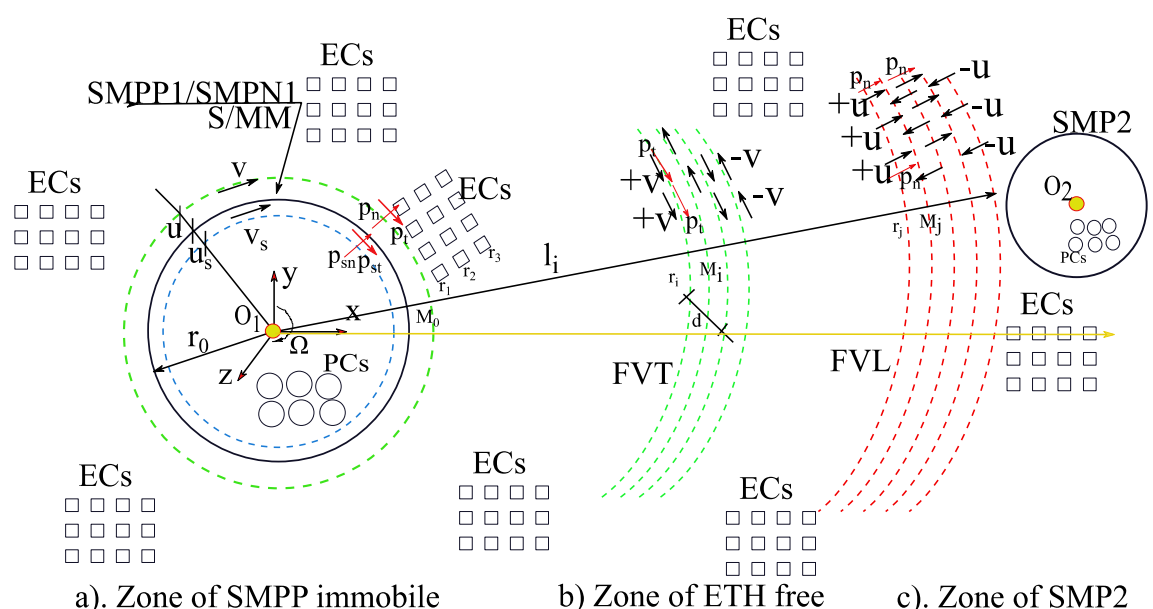

Figure 2. Simplified spherical model of an SMP showing the,$+-u_{s}$ and,$+-V_{s}$ vibrations at its surface and the,$+-u$ and,$+-v$ fundamental vibrations in the ether. 
ECs, due to the periodic oscillatory of normal deformations,$+-u_{s}$ of $S / M M$; these have frequency $f_{n}$ ( $T_{n}$ period) and are mechanically coupled with the rotation speed $\Omega$ of $S / M M$ with frequency $f_{t}\left(T_{t}\right.$ period), will follow (Figure 2).

During the first time interval $\Delta t_{a}=T_{n} / 2$, referred to here as the Active phase (PHA), viz. when SMP acts upon ETH, as long as the deformation by expansion $+u_{s}$ (of $S / M M$ ) lasts, an active attachment appears between SMP1 and ETH, and a tangential elastic clockwise deformation $+v$ is produced by SMP, in the first layers of the ECs in the ETH, due to the rotation of the SMPP with rotation speed $+\Omega$. In PHA, the SMP is active and transmits an active tangential percussion $p_{t a}$ to ETH together with a tangential deformation $+v$, involving mechanical active work:

$$
L_{1}=p_{t a} v
$$

As a reaction to the deformation $+V$, a tangential elastic deformation $-V_{s}$ will also appear counterclockwise in the first layers of the $S / M M$, where:

$$
v_{s}=v
$$

In the following period $\Delta t_{r}=T_{n} / 2$, referred to here as the Reactive phase (PHR), viz. when ETH acts upon SMP as long as the deformation by contraction $-u_{s}$ (of $S / M M$ ) lasts, a reactive deformation appears between SMP1 and ETH, and a tangential elastic counterclockwise deformation $-v$ is produced (as recovery of $+v$ ) in the first layers of the ECs in ETH, concomitant with the rotation of the SMPP with a period $T_{n}$ (speed $+\Omega$ ), (Figure 2). In PHR, the ETH is active, and transmits a reactive percussion $p_{t r}$ to the SMP together with a deformation $-V$, involving mechanical active work:

$$
L_{2}=p_{t r} v
$$

Under stationary conditions of the couple SMP/ETH, the percussion forces will be equal, i.e. $p_{t r}=p_{t a}$, (Newton's $3^{\text {rd }}$ law) and from (1b), (1c) and (1d), this results in a equality in the works done:

$$
L_{2}=L_{1}
$$

Hence, no loss of energy occurs. The energy accumulated in ETH during PHA is entirely returned to the SMP in PHR.

We note that between PHA and PHR when manifests strong adherence between SMP/ETH, there may be a certain period of time $\Delta t_{s}$ when adherence disappears, in which slipping occurs between them, without effort $\tau$ or deformation $V$, in order to allow for a free rotation speed $+\Omega$ of SMP.

The process of periodic transversal deformations,$+-v$ of ETH completed with $\Delta t_{s}$ of slipping SMP/ETH, will be continuous, with normal $T_{n}$ periods:

$$
T_{n}=\Delta t_{a}+\Delta t_{r}+\Delta t_{s}
$$

According to Newton's third law and the MIUP principle from Section 2.1, any deformations,$+-v$ in the ECs from the first few rows $(1,2,3, \ldots)$ of the adjacent ETH will correspond to the pairs of deformations,$+-V_{s}$ from $S / M M$ of the SMP (Figure $2(\mathrm{a})$ ). But this,$+-V_{s}$ is only a partial deformation of $S / M M$, pro- 
duced between slipping times $\Delta t_{s}$, when in a slipping time the rest of a complete rotation displacement $V_{\text {slip }}$, of $S / M M$ sphere takes place by slipping.

We note that PHA/PHR phases in case of a SMPN are offset with $T_{n} / 2$ compared to SMPP.

The frequency $f_{n}$ of the PHA and PHR processes with period $T_{n}$ from Equation (2a), and the size of the deformations,$+-V_{s}$ at the $S / M M$ surface, must be a function of the nature of the contact surfaces, the speed of sliding between $S / M M$ and the surrounding ECs, and of the rotation speed/frequency $\Omega\left(f_{t}\right)$ which are as yet unknown.

However, in the present analysis, it is not strictly necessary to know the sizes of these parameters, and it is sufficient to assume the existence of the deformations,$+-v$ in the ETH, which create FVTs. We will assume that $f_{t} /(\Omega)$ and $f_{n}$ are correlated as whole multiples, depending of number of protuberances in the SMP (Figure 1), including the case where $f_{n}=f_{t}$, for a sphere. In the general case, the vibrations FVL and FVT will have a frequency of integer multiples between them, resulting in:

$$
f_{t}=n f_{n} \text { or } T_{n}=n T_{t}
$$

but in the following analyse we assume for simplicity $n=1$, so $f_{n}=f_{t}$.

Hence, $u_{s}$ and $v_{s}$ type vibrations of SMP will result in periodic deformations ,$+-u$ and,$+-v$ in the ETH. These deformations of longitudinal type $u$ and transversal type $v$, will give rise to permanent vibrations of the ECs in ETH. These vibratory deformations,$+-u$ and,$+-v$, are referred to as FVs in HM16, since they are permanent in time and are uninterrupted by the vibratory deformations in the overall ETH, starting from each SMP1 and reaching or exceeding every existing SMP2 (Figure 2).

FVs may be of two types, corresponding to,$+-u$ and,$+-V$ deformations, and these are referred to as FVLs and FVTs, respectively. Also the lasts may be of two types, corresponding to $\mathrm{P}$ and $\mathrm{N}$ electric sign, as will follow.

\subsubsection{Energy Equilibrium and Transmission}

We now note that at any intermediate point $M_{i}$ on the front surface of an FV wave (Figure 2), each active (initial, from PHA) percussion force $p_{p}$ created by an active initial EC in the ETH will correspond a reactive (secondary, from PHR) force $p_{p}^{\prime}$ (Figure $3(\mathrm{a})$ ).

These percussions $p_{p}^{\prime}$ will be created by the EC' located on the opposite side of the FV front of the vibratory wave, $u$ and $v$ and respectively, of FVL/FVT.

In a state of static equilibrium we have the equality:

$$
p_{p}=p_{p}^{\prime}
$$

The situation for any string $\mathrm{ST} / I_{i}$ or line/path through the vibratory ECs in ETH with $p_{p} / p_{p}^{\prime}$ percussion pairs is shown in Figure 3(a).

A theoretical model of the ECs of the ETH is shown in Figure 3(b), in which these are represented by a string of harmonic oscillators of mass $m$, connected with elastic springs. 


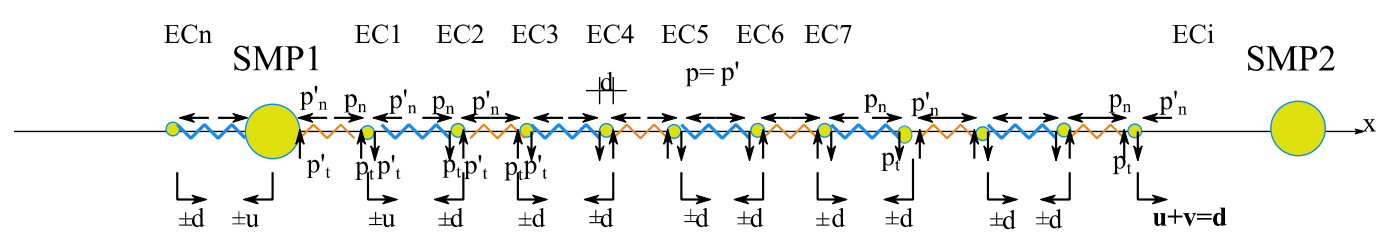

a). Situation of a series of ECs + SMP1 oscillators in ETH in permanent equilibrium of percutions $p_{n}$ and $p_{t}$ with $\mathbf{d}$ total deformation, without energy transport

Oscillators in rest In permanent oscillation

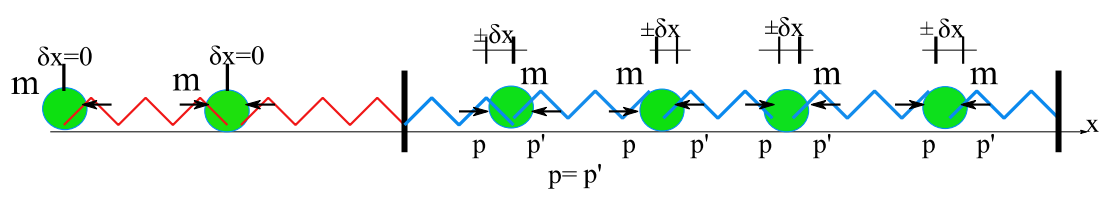

b). Situation of a series of harmonic oscillators from mechanics, in permanent equilibrium of force percutions $p$ With $\delta \mathrm{x}$ deformation, without energy transport

Figure 3. Simplified mechanical model of FVs in a string of ECs in the ETH, without energy loss, as a series of harmonic oscillators.

We notice that the percussion force $p_{p}$ can be transmitted between two SMPs, in a step-by-step manner via a direct string $\mathrm{ST}_{0}$ of ECs arranged on a straight line $\mathrm{O}_{1}-\mathrm{O}_{2}$ between them, through the ETH (Figure 2 and Figure 3), as well as via other less direct paths or strings, according to [8].

We admit in HFVI that at a time $t_{0}$ in the initial periods of the existence of SMP1, considered to be mobile with speed $V_{1}$, and of SMP2, which is mobile with a speed $V_{2}$, in the area/zone of influence (AI) of SMP1 (Figure 2 and Figure 3), the $p_{p}^{\prime}$ reactive force will be less than the active force $p_{p}$ when mechanical work $L$ will be consumed during deformation with $u$ or $v$ (or with the resulting deformation $d$ ) of the ECs, due to the non-zero difference in forces $p_{p}-p_{p}^{\prime} \neq 0$.

$$
L=\left(p_{p}-p_{p}^{\prime}\right) d=E
$$

The mechanical work consumed, $L$, represents a transfer of energy $E$ between the active cell EC and the reactive cell EC', and this process will eventually lead to an transfer of energy $E$ between SMP1 and SMP2 along the $I_{i}$ path or between SMP1 and the ETH ether in the AI, fulfilling the condition $p_{p} \neq p_{p}^{\prime}$.

We note that this transfer of energy $E$ can take place between SMP1 and SMP2 in any direction, along various indirect paths between them, $l_{i}$.

The energy $E$ that corresponds to this mechanical work $L$ will come from the potential energy $U$ created by the percussion-type interaction forces $p_{p}$, which in fact represent the corrected Coulomb-type forces $F_{\mathrm{CC}}$ with which the SMP1 and SMP2 assembly (Figure 2) was originally endowed at the time $t_{0}$ of their creation or stabilization. 
We will now consider the situation of the point $M_{i}$ in the ETH at a later time $t_{1}>t_{0}$, when a state of equilibrium between the active $p_{p}$ percussion forces and the reactive $p_{p}^{\prime}$ force has been reached, thus fulfilling the condition $p_{p}^{\prime}=p_{p}$ along the entire path $I_{i}$ (Figure 2 and Figure 3 ). As a result, at time $t_{1}$, no more mechanical work $L$ will be consumed/developed at point $M_{i}$ on the surface of FV' contact front, between $p_{p}^{\prime}$ and $p_{p}$. This is because the two values of the afferent mechanical work $L$ and $L^{\prime}$ will be equal according to Equation (2a), due to the equality of the forces $p_{p}^{\prime}=p_{p}$ and to the identical movements with $u$ or $v$ (or $d$ ) of their points of application.

This will occur when all ECs in the ETH in the area of any point $M_{i}$ achieve a state of vibration with permanent FVs over time, via,$+-u$ and,$+-V$ deformations, the size of which has become permanent over time (Figure 2 and Figure 3). This can occur when all SMPs in the area reach an immobile state, i.e. they have speed $V=0$. In this way, the percussion forces will be stabilised at a constant value $p_{p}^{\prime}=p_{p}$, shortly after they reach speed $V=0$; however, the oscillation deformation,$+-u$ and,$+-v$ of the ECs remain non-zero and are preserved.

Since the ETH has ideal properties, without friction or other forms of energy loss, there will be no further remote transmission/transfer of energy $E$ between SMP1 and SMP2 in the situation where $p_{p}^{\prime}=p_{p}$ (Figure 3).

In the case of static and dynamic equilibrium between the specific percussion forces,$+-p_{n}$ and,$+-p_{t}$ (Figure 2 and Figure 3), no energy transmission from SMP1 to SMP2 occurs via the ECs of ETH. The initial/stabilised energy of SMP1 will be preserved, although the particle will continue to transmit FVLs and FVTs continuously over time. However, these FVs will no longer absorb energy from $\mathrm{SMP}_{0}$, meaning that they will no longer transmit energy through the ETH during operation under these conditions for SMPs that are immobile and have reached static and energy equilibrium.

This is because in the case of static equilibrium, the specific percussion forces ,$+-p_{n}$ and,$+-p_{p}$ will act symmetrically both ways at their contact point $M_{i}$ : As a result, an energy balance situation is reached in the ETH in the AI zone (Figure 2 and Figure 3 ).

The majority of the ETH in nature is in this situation of energy equilibrium, in AI areas, free from SMPs or far from any SMP moving at a speed $V \neq 0$.

The physical matter in the universe, consisting of SMPs, is only in this state of energy balance and static equilibrium between percussion forces $p_{p}$ if these SMPs are in a motionless state so with $V=0$, or at a finite time interval, after the creation of these SMPs (if created).

This results in the case discussed in this Section above, where an energy $E / L$ balance can be achieved under certain conditions in the process of emission/reception of FVs by an SMP1. These FVs can be continuously emitted over an indefinite time by any SMP1 in equilibrium, without consuming the SMP1's own energy. We can therefore conclude the following:

1) We are justified in assuming in the HFVI that in the ETH, within an energy balance AI zone, due to the creation at a given point $M$ on $S / M M$ surface, of pe- 
riodic spherical forces (balloon type) of normal $+/-p_{s n}$ percussions of tensile/compression $+/-\sigma$ type, a series of linear $+/-u / \varepsilon$ deformations, will be created throughout the surrounding ETH. And that these,$+-u$ are transmitted in the form of longitudinal spherical vibrations (FVLs) that are permanent over time, at energy equilibrium (Figure 2).

2) In the event of the appearance of periodic transversal/circular $+/-p_{s t}$ percussion forces of the,$+-\tau_{s}$ shear type at point $M$ on a spherical surface $S / M M$ with vibration/rotation axis $\mathrm{O}_{1} Z$, periodic transversal/angular deformations ,$+-v / \gamma$ will be created throughout the ETH, which shall be transmitted in the form of transversal spherical FVT vibrations, permanent over time, at energy equilibrium (Figure 2).

However, we admit that the vibratory deformations,$+-u$ and,$+-v$ in ETH can be transmitted by any SMP1 via periodic,$+-p_{p}$ percussion forces, from one EC to another in the form of a cell string $\left(\mathrm{CS}_{\mathrm{i}}\right)$ (Figure 2 and Figure 3), and deformations occur in the form of FVL and FVT vibrations.

We do not currently have concrete data on the sizes of the propagation velocities $c_{f L}$ and $c_{f T}$ of the FVLs and FVTs, respectively, or on the sizes of their frequencies, $f_{f L}$ and $f_{f I}$, however, these are not strictly necessary in our current analysis.

\subsection{Physical Nature of electrical Charges Due to PVP/PVN Vibrations of SMPs in HFVI}

Based on experience, we can assume in HFVI that SMPs must be differentiated firstly in terms of their electrical aspect, currently considered in physics to be given by an electrical charge $+q /-q$ that is intimately attached to an SMP, although this $+q /-q$ charge has not been defined physically, as substance.

This electrical aspect of SMPs is physically manifested in specific ways that can be distinguished in experiments, i.e. SMPP and SMPN (denoted in [1] as $\mathrm{SMP}^{+}$and $\mathrm{SMP}^{-}$, respectively).

We also assume the existence of neutral SMPNEs, including neutrons, but we admit that these are also composed of particles of types SMPP and SMPN. Inside an SMPNE, these SMPPs and SMPNs are grouped into neutral minidipoles (MDs) with zero electrical charge (Figure 1(b)), resulting in a total electrical charge of zero.

We can verify this structure of the SMPNE neutron. For this, we assume that a neutron has a diameter 10 times as large as an electron/positron. It therefore comprises approximately $10^{3}$ such MDs, consisting of electron/positron doublets (whose arm $b$ is about 10 times smaller than for atomic dipoles) (Figure 1(b)). The mass of a neutron results about $2 \times 10^{3}$ times the mass of an electron $m_{e}$ as the currently accepted mass of the proton/neutron is close to $1840 \mathrm{~m}_{e}$. Hence, electron/positron doublets within the neutron represent an organised accumulation of highly compacted MD doublets, which in the presence of an adequate energy input $\Delta E$ can release an electron or positron; when a single electron is released, the neutron becomes a proton. A proton has the same composition as a 
neutron, but possesses an extra positron (or equivalently, lacks an electron).

This electrical differentiation of the observable, external manifestation of SMPPs and SMPNs, must originate in the specific internal modes of vibration of the internal PCs, from the PVs of the PR protoplasm, including the vibration/rotation mode with rotation speed $\omega$ of the PCs, which constitutes the external membrane MM. These PCs are also particles of an ethereal nature but have their own composition that is different from ECs; this is likely to incorporate a large number of ECs, actually constituting a series of quark-type SSMPs.

Thus, we postulate in HFVI that there will be a system of internal vibration/rotation PVs of PCs in the positive SMPP, denoted as PVP (particle vibration positive); an internal vibration system for SMPN PCs, denoted as PVN; and for SMPNEs, denoted as PVNE.

Next, we assume that the primary difference between vibration modes, PVP and PVN, of SMPs lies in the sense of the rotation of the $S / M M$ surface/membrane with the rotation speed $+/-\Omega$ around the $\mathrm{O} z$ axis (Figure 2).

We assign the clockwise sense $(+\Omega)$ of rotation around the $\mathrm{O} z$ axis to the rotation mode of the PC from the MM of the SMP denoted by PVP, and the anti-clockwise rotation direction $(-\Omega)$ for the rotation mode of the PC cell from the MM of SMP, denoted by PVN.

The PVP and PVN modes arise on the outside $S / M M$ rotations $+/-\Omega$, of the SMP superposed with the,$+-p_{s n}$ and,$+-p_{s t}$ percussions, which are associated to the deformations,$+-u_{s}$ and,$+-v_{s}$ on the outer surface $S$ of the SMP (i.e. the $M M)$. And then PVP/PVN modes, manifest through the percussions,$+-p_{n}$ and ,$+-p_{t}$ associated to deformations,$+-u$ and,$+-v$ of ETH cells ECs, as discussed in Sections 2.6 and 4 .

The,$+-u$ and,$+-v$ deformations in ETH will give rise to the FVs of the ECs of the ETH external to SMP1. The two types of particle, SMPP and SMPN, will appear on the outside ETH, by their specific FVs, and are differentiated based on the effect that is referred to today as electric, but in reality arises from the specific FVs created in the ETH (Figure 2).

In the following, we will denote the FVLs/FVTs produced by the positive particles (+/P) SMPP as FVLP/FVTP, respectively; we will denote the FVLs/FVTs produced by negative particles $(-/ \mathrm{N})$ SMPN as FVLN/FVTN, and the vibrations of neutral particles as FVLNE/FVTNE.

\subsection{Creation of a Pair of SMPs}

In order to obtain information on how to differentiate between the vibrations produced by SMPPs and SMPNs, we can analyse the initial phase of creation of these SMPs according to HFVI.

We assume that the creation of SMPPs and SMPNs is a phenomenon similar to the creation of electron/positron pairs by hard electromagnetic (E-M) $\gamma$ waves. We assume that the starting point for the creation of SMPs is at the origin $\mathrm{O}$ of a Cartesian RF Oxyz that is fixed in the ETH (Figure 4). 


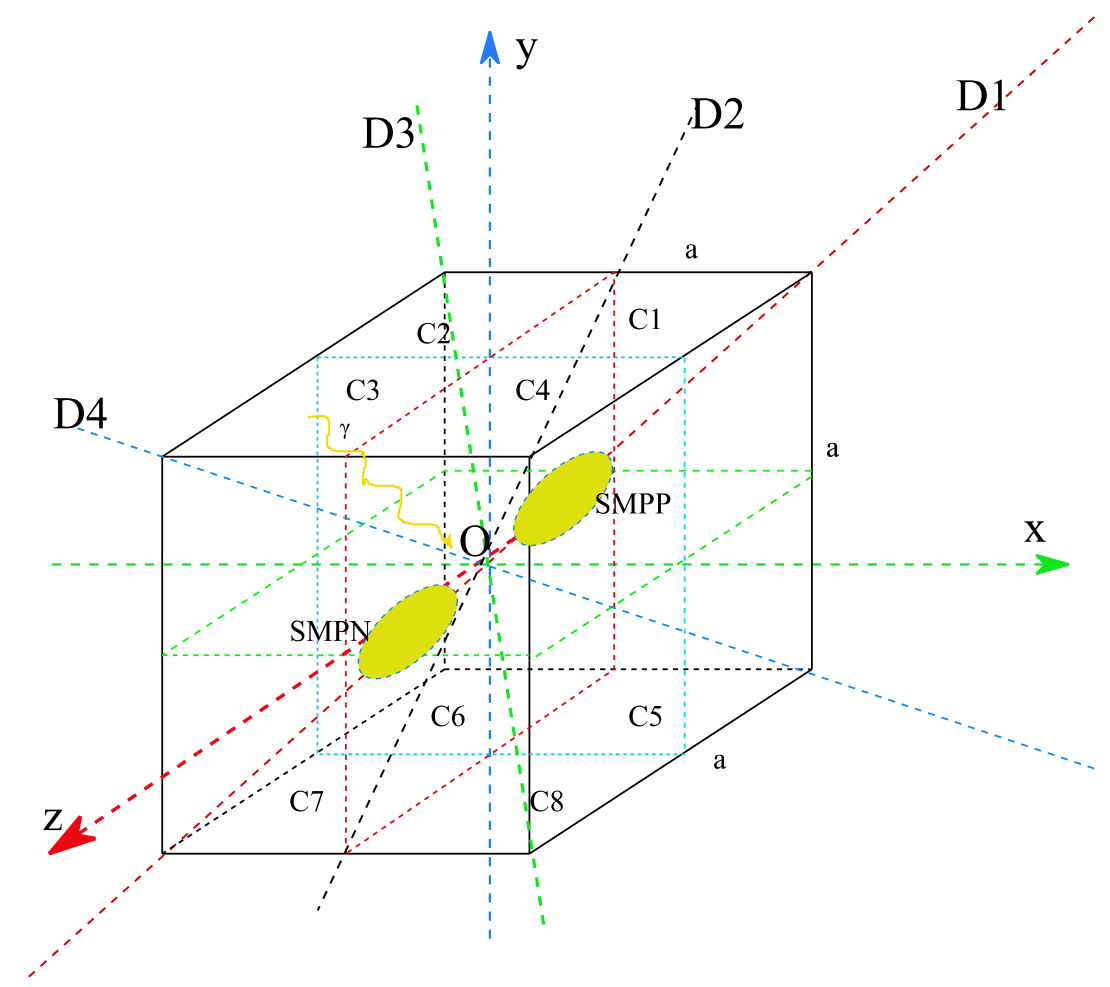

Figure 4. The Oxyz reference frame at the centre of a cube with sides a, diagonals $D_{1}-D_{4}$, and quadrants $\mathrm{C}_{1}-\mathrm{C}_{8}$ at the time of creation of an SMPP/SMPN pair.

Immediately after its creation, the SMPP will be located in the $\mathrm{C}_{1}$ quadrant of $\mathrm{RF}$, and the SMPN will simultaneously be located symmetrically from the origin $\mathrm{O}$, along the $\mathrm{D}_{1}$ diagonal of the RF, meaning that it is in quadrant $\mathrm{C}_{7}$ (Figure 4). The SMPP and SMPN are shown here in a simplified form, immediately after their creation, by two ellipsoids aligned along diagonal $\mathrm{D}_{1}$.

Let us now look at the likely deployment of the creation of the two particles, SMPP and SMPN, by a PH photon with total energy $E_{p h}$ of the radiation $\gamma$ order and with size smaller than an SMP, but of the same order of magnitude.

Initially, at point $\mathrm{O}$, within an area close to an MPP/MPN pair of microparticles (not shown), the photon PH begins to develop spatially within the ETH. $\mathrm{PH}$ will release its $E_{p h}$ energy into the ETH, and as a result, a series of vortices (VOs) will occur in ETH, comprising the first ECs within the surrounding ETH. These ECs cells will become groups of particle cells (GPCs), and will also take up an amount $\Delta E$ of the energy $E_{p h}$.

This group of VOs undergoes rapid expansion over the neighbouring ETH, initially creating an expanding bubble $\mathrm{BU}_{0}$ around $\mathrm{PH}$ made up of all the newly formed VOs, grouped into new GPCs. However, within a short period, $\mathrm{BU}_{0}$ will tend to divide to give two new SMPs. Thus, $\mathrm{BU}_{0}$ becomes elongated in the direction of the diagonal $\mathrm{D}_{\mathrm{i}}$ of the $\mathrm{Oxyz}$ reference frame (Figure 4). In the opposite quadrants $\mathrm{C}_{1}$ and $\mathrm{C}_{7}$, two ellipsoids $\mathrm{EL}_{1}$ and $\mathrm{EL}_{2}$ emerge from $\mathrm{BU}_{0}$; these are at first united by a thick common area that rapidly thins, and towards the end forms a connecting cord $\mathrm{CO}_{0}$ (Figure 4). This cord rapidly thins, becoming a 
single CS string of ECs, and finally breaks completely.

Thus, in the two quadrants I and VII, the two SMPP and SMPN are formed from $\mathrm{EL}_{1}$ and $\mathrm{EL}_{2}$, due to which we can admit that there is a differentiation in the shape of the two spherical/symmetrical surfaces $S$, given by the last stage of the $\mathrm{CO}_{0}$ break. A flaring will arises in the SMPP remaining from $\mathrm{CO}_{0}$, and in the SMPN, a deepening produced by $\mathrm{CO}_{0}$ will arise; however, these differentiations are equivalent as volume of ether and these differentiations makes sense to exist.

These differentiations between the SMPP and SMPPN arise from the start of their formation as embryo ELs as new autonomous SMPAC cells (Section 1), when a nucleus NC structure was created for the positive (DNAP) and negative (DNAN) particle types. Due to their specific ADNs, these NCs are differentiated based on their future electrical charge-type behaviours, resulting in the positive SMPP and the negative SMPN, as described in Section 2.4.

We also postulate in HFVI that during the formation and growth of the original two ELs, an equilibrium is created between the volume of ECs cells embedded in any EL and the number of CEs taken from the ETH, meaning that there is no change in the volume of the $\mathrm{ETH}$ in the $\mathrm{BU}_{0}$ zone from the creation of the SMPP and SMPN, and no change in the natural pressure of the ETH in the area.

We postulate in HFVI that at the time of breaking of the cord $\mathrm{CO}_{0}$, it is in a state of tension $(+\sigma)$ that opposes the break-up of the SMPP and SMPN. We also assume that within both the $\mathrm{CO}_{0}$ cord and the ELs, and also in the new SMPP and SMPN, internal rotations/vortices PV of the particles, with rotation speed $\omega$, arise to maintain the energy reserve $E$ of $\mathrm{PH}$, in similar way to other SMPs. This energy reserve $E$ allows for the creation and distancing of the two particles, SMPP and SMPN, with a certain initial speed $V_{0}$. After reaching an equilibrium speed $V_{e q}$ or $V=0$, the SMPP/SMPN system will possess a potential energy $U$ that is equivalent to the kinetic energy $E_{k i n}$ at the initial time of expulsion of the particles; this energy ensures normal operating conditions for the system in the ETH.

At a later time, the two particles, SMPP and SMPN, will be able to approach again, and will tend to reunite to give the original form of the photon $\mathrm{PH}$ through the annihilation process (the inverse of the process described above in this Section). The proximity needed for this reunion can be achieved by an interaction force $+F_{L}$ from the attraction between the SMPP and SMPN, produced by periodic percussions,$+-p_{p}$ that are created by each particle in the ETH by FVLs (as discussed in Section 3).

The force $+F_{L}$ will also arise as described in Section 1, for SMPAC and HCO, through the specific attraction orders provided by the NCs of the SMPP and SMPN. In addition,,$+-p_{p}$ percussions are generated from the FVLs created by each type of SMP: FVLP or FVLN, depending on the electrical sign $(+/ \mathrm{P}$ or $-/ \mathrm{N})$ of the SMP respectively.

When a SMPP and SMPN pair regroups (Figure 4), a new $\mathrm{CO}_{0}$ cord will be created between them, representing an initial $\mathrm{CO}_{0}$ reproduction; this will initial- 
ly consist of a $\mathrm{CS}_{0}$ string of ECs, and will regain its initial stretching effort $(+\sigma)$, created by permanent percussions,$+-p_{p}$, each of which exerts a reciprocal force of attraction $+F_{L}$ between SMPP and SMPN, as described in Section 3 .

However, when grouping a pair of two identic signs SMPs, a new $\mathrm{CO}_{0}$ cord will be created between the two particles. This will also now gain a compression effort created by permanent percussions,$+-p_{p}(-\sigma)$, thereby exerting a mutual force of rejection $-F_{L}$ between the two particle, which both have the same electrical nature, as they have the same sign, as discussed in Section 2.4. This physical manifestation is confirmed by the presence of Coulomb-type forces, with the charges $+q$ and $+q$ from electrostatics.

A more detailed mechanism for the process of interaction between SMPs via forces $F_{L}$, based on percussion forces,$+-p_{p}$, will be presented in Section 3 .

We note that this mode of interaction between two SMPs is also supported by the fact that it corresponds to a manifestation in the ETH of the dialectical principle of attraction of the opposites. This principle is also found in nature in the process of reproduction of living beings (and also in inanimate world in some situations), for example the attraction between different sexes; this is spontaneous and natural, since most animals can reproduce only by combining two types of DNA. The reverse of this principle, manifested by the natural revulsion between identical sexes, which have only one type of DNA, also holds true, and this is also manifested in the case of SMPs, as described above.

\subsection{Specific Action Modes of SMPP/SMPN on ETH, for Creating the four Types of FVs}

\section{1) SMPP with FVLP:}

We postulate in HFVI that an SMPP which produces FVLs, is in a permanent state of energy balance with the external ETH, as discussed in Section 2.3.2

Then, radial/linear deformations,$+-u_{s}$ at its $S / M M$ surface, with spherical symmetry, starts at some time $t_{0}$, through a positive dilation $+u_{s}$ that corresponds to an increase in radius $r_{0}$, and this gives rise to an expulsion of a volume of ETH corresponding to $+u_{s}$ (mechanism is presented in Section 3.). This initial dilation $+u_{s}$ will be followed by a contraction $-u_{s}$ in $S / M M$, so that an absorption of the ETH corresponding to $-u_{s}$ is produced (Figure 2). A new cycle will follow over a period $T_{L}$ of dilation/contraction of $S / M M$ with,$+-u_{s}$ deformations with frequency $f_{L}$, etc.

Thus, the total variation in the radius $r_{0}$ of the SMPP over one complete vibration will be,$+-u_{s}$ of amplitude $1 / 2 u_{s}$, which corresponds to an average expulsion of ETH of $+1 / 2 u_{s}$. The maximum deformations,$+-u_{s}$ will be periodic, and thus vibratory, giving rise in ETH to FVLPs with frequency $f_{L}$ or period $T_{L}$.

This is the specific manifestation of SMPP considering the creation in ETH of ,$+-u$ deformations with spherical symmetry, which will produce FVLPs in whole ETH.

2) SMPN with FVLN: 
We also postulate in HFVI that in the case of an SMPN, which produces FVLs, the phenomenon is symmetrical to the case described in point 1) for the SMPP, including spherical symmetry of deformations, as shown in Figure 2. At the initial time $t_{0}$, there will be an initial contraction deformation of size $-u_{s}$, at the surface $S / M M$, corresponding to the negative direction of the radius vector $r_{0}$, and hence also an initial absorption of a volume of ETH corresponding to $-u_{s}$, thus offsetting the deformation $+u_{s}$ for the SMPP from point 1). This initial contraction will be followed by a dilation/growth of $r_{0}$ on $S / M M$ with $+u_{s}$. A new cycle of contraction/dilation of $r_{0}$ on $S$ will follow with,$+-u_{s}$ deformations etc. (Figure 2). Thus, the total variation in the radius $r_{0}$ of the SMPN in a complete vibration with frequency $f_{L}$ will be $-u_{s}$, with amplitude $1 / 2 u_{s}$, corresponding to an average absorption of $-1 / 2 u_{s}$ of ETH and maximum deformation,$-+u_{s}$, giving rise in ETH to FVLNs with frequency $f_{\mathrm{L}}$ or period $T_{L}$.

This is the specific manifestation of SMPN considering the creation in ETH, of,$+-u$ deformations with spherical symmetry, which will produce FVLNs in whole ETH

But this contraction of $-u_{s}$ in $r_{0}$ in the SMPN, occurs exactly during the period of dilation by $+u_{s}$ from the corresponding SMPP (Figure 2). The two deformations $+u_{s}$ and $-u_{s}$ in the SMPP and SMPN are therefore in phase opposition, and the expulsion of ETH by the SMPP corresponds in time to the absorption of ETH by SMPN.

After each period $T_{L}$, because the directions of deformation,$+-u_{s}$ are opposed ,$-+u_{s}$, thus preserving the volume of the ETH, by ensuring a null variation in its volume $\left(\Delta V_{o l}=0\right)$, thus maintaining constant the volume of ETH in the space around the SMPP and SMPN pair (Figure 2). This is similar to the phenomenon of liquid compensation between two interconnecting vessels, and can explain the observation in electrostatics of the cancellation of the total charge around two charges $+q$ and $-q$ that are close one to another. This is also the specific way in which an electric DI dipole is manifested through FVL.

\section{3) SMPP with FVTP:}

We also postulate in HFVI the case of an SMPP having the rotation speed $+\Omega$, which will be the source of FVTP produced by ETH deformations,$+-v$.

But deformations,$+-V$ will be created by equivalent tangential/transversal deformations,$+-V_{s}$ in its $S / M M$ surface, with cylindrical symmetry around the $\mathrm{O} z$ axis. These equivalent deformations,$+-V_{s}$, are special, being even those which, if there were real, would create in the ETH the vibrations deformations,$+-V$, with $v_{s}=v$.

These equivalent deformations,$+-V_{s}$ are due to rotation speed $+\Omega$ of SMPP, produced by successive adhesion and sliding between $S / M M$ and the adjacent ECs in ETH, according to the mechanism mentioned in Section 2.4 and described in Section 4. More formally, the deformations,$+-V_{s}$ correspond to alternating partial rotations of $S / M M$ around its own axis $\mathrm{O} z$, starting at a given time $t_{0}$ (Figure 2). These equivalent deformations in the SMPP are periodic with fre- 
quency $f_{t}$ (or rotation speed $+\Omega$ ) around the Oz axis of the SMP, or period $T_{t}$.

In the case of the SMPP, we assume that the initial equivalent tangential deformation at $t_{0}$ is $+V_{s}$, a clockwise rotation (denoted here as positive), followed by a counterclockwise rotation $-V_{\mathcal{S}}$, then by a rotation $+V_{s}$, and so on (Figure 2). All rotation deformations,$+-V_{s}$, have the frequency $f_{t}$ or period $T_{t}$ and involve oscillating rotation of the radius $r_{0}$ of the SMPP around the $\mathrm{O} z$ axis.

This is the specific manifestation of SMPP considering the creation in ETH of tangential,$+-V$ deformations with cylindrical symmetry around the $\mathrm{Oz}$ axis, which will produce FVTPs in whole ETH as at point 5).

\section{4) SMPN with FVTN:}

In the case of the SMPN, which produces FVTNs, we assume that the initial rotation equivalent deformation is $-v_{s}$ counterclockwise (negative), followed by a clockwise rotation $+v_{s}$, then by a rotation $-v_{s}$, and so on, with cylindrical symmetry around the $\mathrm{O} z$ axis. All rotations have frequency $f_{t}$ and involve deformations $-V_{s}$ corresponding to a counterclockwise rotation of the radius $r_{0}$ of the SMPN around the $\mathrm{O} z$ axis, giving rise to vibrations (Figure 2). These deformations,$-+V_{s}$ are actually periodic grips/slips between $S / M M$ and the ETH (similar to the fault slippages that create transversal waves in earthquakes).

This is the specific manifestation of SMPN considering the creation in ETH of ,$-+V$ deformations with cylindrical symmetry around the $\mathrm{Oz}$ axis, which will produce FVTNs in whole ETH, as at point 5).

Therefore here we postulate in HFVI that these tangential equivalent deformations at time $t_{0}$ (i.e. the first $+v_{s}$ in the SMPP and the first $-v_{s}$ in the SMPN) are in opposition (i.e. out of phase) in terms of the sense of rotation (Figure 2). This opposition between the senses of rotation $+v_{s}$ and $-v_{s}$ creates compensation (constancy) between the kinetic moments $J$ of the SMPP and SMPN pair, starting at the moment $t_{0}$ of their creation. There is then no need to transfer the kinetic moment $J$, according to Newton's third law, to the ETH external from the SMPP and SMPN pair, as the mechanism described above and in Sec. 4 is in effect. At the same time, the change in volume of the ETH due to,$+-\gamma$ will be compensated.

5) Consequences of ETH deformations produced by SMPs:

We also postulate in HFVI that these periodic deformations,$+-u_{s}$ and,$+-V_{s}$, which produce at frequencies $f_{L}, f_{T}$, on the surface $S / M M$ of the SMP, will be immediately transmitted to the surrounding ETH in the form of periodic external deformations,$+-u$ and,$+-V$ (Figure 2). These produce FVLs and FVTs in the ETH, respectively, due to the mechanism discussed above, and in Sections $2.4,3$ and 4 , respectively.

We also assume in HFVI that these periodic deformations,$+-u_{s}$ and,$+-v_{s}$ in the surface of an SMP, as well as the deformations,$+-u$ and,$+-v$ in the ETH, are deformations/displacements with minimum intrinsic/predetermined size in both SMPs and ECs, and represent the minimum steps or displacements $\Delta x$ in quantum mechanics. 


\section{Application of HFVI to the Interaction between Immovable SMPs in the Case of FVLs}

\subsection{Interaction between Two Positive Immovable SMPPs through FVLS}

\section{1) General conditions of SMPPs:}

In some SMPPs, vibrational deformations,$+-u_{s}$ will appear on the surface $\mathcal{S}$, and physically on the membrane MM, as explained in Section 2.6.(a) (Figure 2). These radial deformations,$+-u_{s}$ are spherically distributed on $S / M M$, and are completely symmetrical to a point.

Figure 5(a) shows a section through a pair of particles SMPP1 and SMPP2 that are fixed in the $\mathrm{O}_{1} \mathrm{xyz}$ reference frame attached to SMPP1, which is considered immovable in the ETH.

Figure 5(a) also shows the vibratory deformation vectors,$+-u_{s}$ (radial) for SMPP1 and the $S / M M$ surface/membrane of SMPP2 (the first with size $+u_{s}$ and the remainder with size,$-+u_{s}$ ) and the vibratory deformation vectors,$+-u$ (radial) of the first row of ECs $r_{1}$ in the ETH adjacent to the surfaces of the SMPPs. The vibratory deformations FVLs are created by,$+-u_{s}$ acting on the surface $S / M M$ of the SMPP through percussion forces,$+-p_{s}$ from SMPP on the ECs adjacent to the surface $S / M M$ in the ETH, as described in Sections 2.3.1, 2.4, 2.6, and illustrated in Figure 2.

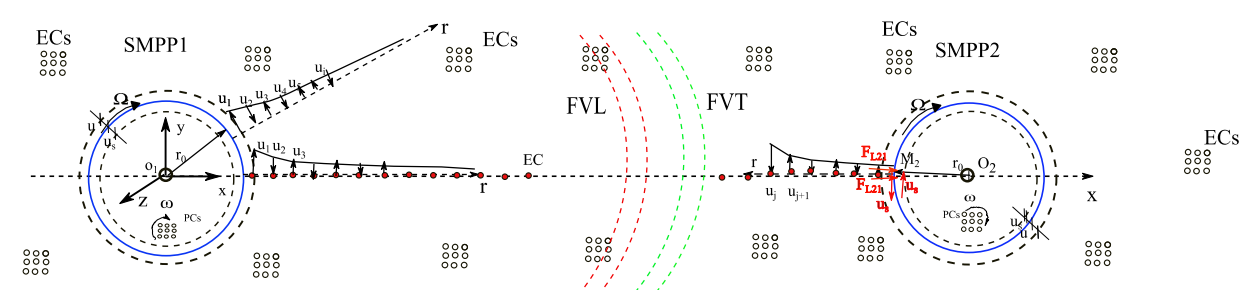

a). Situation of SMPP1/SMPP2 with deformation $u, u_{s}$ and normal forces $F_{L 21}$
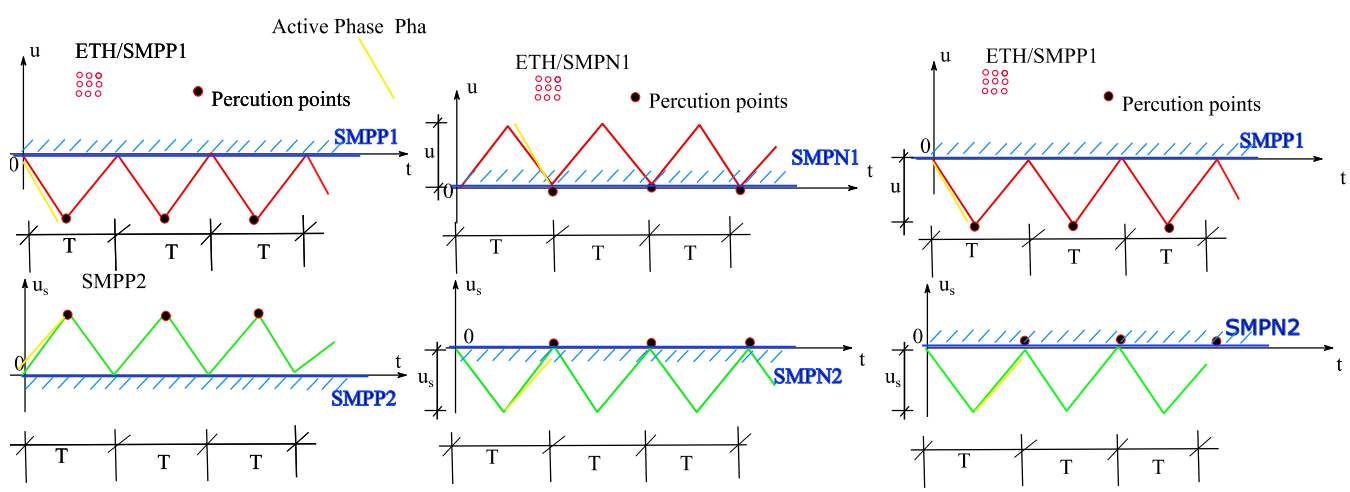

b). Grafic of u deformations in time $t$ and percution points upon SMPP2 from SMPP1 giving $\mathrm{F}$ repulsion forces c). Grafic of $u$ deformations in time $t$ and percution points upon SMPN2 from SMPN1 giving $\mathrm{F}$ repulsion forces d). Grafic of u deformations in time $t$ and percution points upon SMPN2 from SMPP1 giving $\mathrm{F}$ attraction forces

Figure 5. Physical mechanism of the interaction via percussions,$+-p_{p}$, and the evolution of attached deformations $u$ over time, between pairs of SMPs through their FVLs in the ETH. 
The deformations,$+-u$ in the ETH are spherically propagated, since they are completely symmetrical to a point, in all space. The vibratory deformations,$+-u$ induced in the rest of the ETH will be permanently accompanied by related periodic percussions,$-+p_{p}$ (on a single EC) arising from SMPP. We consider that the dilation deformations $+u_{s}$ correspond to the percussion forces $-p_{p}$ in the $\mathrm{ETH}$, since compression is denoted as negative, and vice versa: the deformations $-u_{s}$ correspond to $+p_{p}$, i.e. tensile forces.

The first deformations,$+-u$ are then transmitted, starting from each SMP, step by step via adjacency/contact, in the form of continuous strings (STs) of ECs, and will reach through the other ECs via the surrounding ETH, including from SMPP1 to SMPP2. However, the sizes of these deformations,$+-u$ will be reduced with increasing distance $r$ (Figure 5(a)) corresponding to a variation $1 / r^{2}$ in a similar way to Coulomb's law (as described in Section 6 of [8]).

For clarity, the vibratory deformations,$+-u$ in the rest of the ETH at various distances $r$ are represented by $90^{\circ}$ folds perpendicular to the direction of the ray $r$ by tangent arrows of appropriate size, starting from the surface $S$ of SMPP1 and SMPP2 (Figure 5(a))

Figure 5(a) shows several fronts of action of the vibratory deformations $u$, which are represented as portions of large dotted circles (red for FVLs from an SMPP), as fundamental spherical FVL waves, in the ether ETH, (symbolically represented by rare squares/circles groups, although ECs must in fact be adjacent). Here, we analyse only the direct interaction between SMPPs through a single linear, direct string $\mathrm{ST}_{0}$ (the central one) of ECs cells of radius $r_{00}$ (Figure $5(a))$.

The indirect interaction between two SMPPs via multiple strings $\mathrm{ST}_{\mathrm{i}}$, in the form of a series of progressive curves, and the effect of these strings/paths that create the corrected Coulomb force $F_{\mathrm{CC}}$ as a result, were analysed in [1].

\section{2) Action on SMPP2:}

We will first analyse the case in which the forces produced by SMPP1 in $M_{2}$, the central point on the surface/membrane $S / M M$ of SMPP2, are oriented towards SMPP1 (Figure 5(a)). Periodic linear/longitudinal (here, radial) deformations,$+-u$, the first with size $+u$, and the remainder with size,$-+u$ (associated with,$-+p_{n}$ from SMPP1) near SMPP2, as well as the deformations,$+-u_{s}$ in SMPP2 (associated with,$-+p_{n}$ from SMPP2) permanently act on the surface $S / M M$ of SMPP2, and are shown as dependent on the time $t$ in the detail in Figure 5(b) by a periodic zigzag line (ZL), with a regular period of variation $T=T_{L}$, starting from $t_{0}=0$.

Although the correct real ZL allure may be different from that shown in Figure 5(b) (for example a periodically interrupted curve, lines with different slopes, or even a sinusoid), this detail is not significant in the current analysis.

When the vibratory deformations,$+-u$ from SMPP1 reach point $M_{2}$ on SMPP2 at time $t_{0}$ (Figure 5(a)), we postulate that the phase of the vibration $+u$ from FVLP from SMPP1 is the same phase as the vibration $+u_{s}$ of PVP from SMPP2. In fact, the FVLPs arrive with the same phase at all SMPPs in the ETH, 
due to the theoretically ideal properties of the HM16 ETH, while also respecting the situation of the phases, since the moment of creation of the two SMPs described in Sections 2.4, 2. 5 .

We will analyse the action of SMPP1 on SMPP2 through FVLs, under the general conditions for the behaviour of SMPs in HFVI, described in Sections 2.3.1., 2.6.

At the central point $M_{2}$ on the surface $S / M M$ of SMPP2 facing SMPP1 (Figure 5(a) and Figure 5(b)) we observe that at time $t_{0}=0$, the first deformations $+u$ of the ECs in the adjacent ETH, originating in SMPP1 become $-u$ (due to the reversal of the direction of the $+u$ axis) when applied to SMPP2.

It follows that the deformations,$+-u$ in $\mathrm{ETH}$, which have become,$-+u$ (Figure 5(b)), now have the opposite orientation to the,$+-u_{s}$ deformations in the PC cells in SMPP2, although they have the same phase. The peak points $P_{i}$ of the ZL curve of the periodic,$-+u$ deformations in ETH at various time intervals $T$, where the percussion forces $-p_{n}$ are focused, (on a single EC, as part of PHA active phase, from Sections 2.3.1., 2.4), will be directly opposite to the peak points $\mathrm{P}_{\mathrm{i}}$ of the ZL curve of the,$+-u_{s}$ deformations in SMPP2 (Figure 5(b)). The compressive/active percussion forces $-p_{n}$ are concentrated at these contact points $\mathrm{P}_{\mathrm{i}}$ having opposed senses, so giving repulsion force (Figure 5(b)).

In this case, in point $M_{2}$ on the surface of SMPP2, in the area of a single EC, normal/linear compression percussion forces $-p_{n}$ (negative $-\sigma$ compression effort), will occur, i.e. the repulsion of SMPP2 from SMPP1.

Hence, on the elementary surface $S_{\mathrm{s}}$ (a circle with radius $r_{00}$ ) corresponding to the central string $\mathrm{ST}_{0}$ of ECs, at point $M_{2}$ located on the surface $S / M M$ of SMPP2, a continuous $-p_{\underline{\underline{n}}}$ (underlined) repulsive force will appear as an average over a time $T$ of the periodic percussion forces $-p_{n}$. This periodic force of repulsion upon SMPP2 due to SMPP1, denoted as $-F_{L 21}$, is created by periodic percussions $-p_{n i}$, based on their average effort $-\sigma$, on the elementary surface $S_{\mathrm{s}}$ of an EC:

$$
-F_{L 21}=-p_{n}=S_{s}(-\sigma)
$$

This repelling force $-F_{L 21}$ corresponds to the direction/line $\mathrm{O}_{1}-\mathrm{O}_{2}$ of direct interaction between SMPP1 and SMPP2 through $\mathrm{ST}_{0}$ of line $I_{0}$ (Figure 5(a)).

From Figure 5(a) and Figure 5(b), the nature of $-F_{21}$ (on a single EC) is obvious; this is a mechanical force derived from the compressive percussion forces $-p_{n}$ through their average $-\sigma$ compression efforts, over the area $S_{\mathrm{s}}$ of an EC, according to the mechanism discussed above and illustrated in Figure 5(b). At the same time, it can be seen from Figure 5(a) that the force $-F_{L 21}$ corresponds to the Coulomb force of repulsion $F_{C}$ between two positive charges, $+q /+q$, and that the force $-F_{L 21}$ must vary as $1 / r^{2}$ in the same way as $F_{C}$ at relatively short distances.

$$
-F_{L 21}=f\left(r_{0}, r_{00}, p_{n}, \frac{1}{r^{2}}\right)
$$

The full form of the function $f$ in Equation (7) can be established in future on 
an experimental basis depending on the geometry of the SMPs (radius $r_{0}$ ) and the ECs (radius $r_{00}$ ).

Note 3.1: This correlation between the periodic deformations,$-+u$ of SMPP2 and SMPP1 giving repulsion force $-F_{L 21}$, (on a single EC) is also ensured according to the mechanism described in Section 1 for SMPAC and HCO, through the specific rejection commands provided by the NCs of SMPP1 and SMPP2

Note 3.2: This correlation between the periodic deformations,$-+u$ of SMPP2 and,$+-u$ of SMPP1 giving repulsion force, is also ensured according to the mechanism described in Section 2.3.1., where the percussion forces $-p_{n}$ are focused, as part of superposition of two PHA active phases, having opposed senses, so giving repulsion force.

Note 3.3: We postulate in HFVI that the action at the level of an EC cell of the percussions $-p_{n}$ and the equivalent force $-F_{L 21}$ (on a single EC) in Equation (7) represent forces of intrinsic minimum/predetermined size for the SMPs and ETH, which are exactly same as the minimum forces of action $\Delta F$ in quantum mechanics.

Summary 3.1: We postulate in HFVI that the vibratory deformations,$+-u_{s}$ at the surface $S / M M$ of SMPP1 create VFL vibrations in the ETH with deformations,$+-u$ and associated periodic percussion forces,$+-p_{n}$, (on a single EC) which act upon SMPP2 over the area $S_{\mathrm{s}}$ of $\mathrm{ST}_{0}$, the central string (Figure 5(a)). These deformations,$+-u$ overlap at the maximum points of contact $\mathrm{P}_{\mathrm{i}}$ with the deformations,$+-u_{s}$ at the surface $S$ of SMPP2. The deformations,$+-u$ and,$+-u_{s}$ have opposite phases in terms of their physical direction, belonging to PHA of Section 3.2 (Figure 5(b)). Hence, at the overlapping points, percussion forces $-p_{n}$ will be created with the same sign, where $-p_{n}$ represents compression forces, and thus SMPP2 is repelled by SMPP1 (Figure 5(b)). As a result, periodic percussion forces $-p_{n}$ on SMPP2 will create periodic $-\sigma$ efforts at the surface $S_{s}$ of an EC of radius $r_{00}$, which give an average resultant force $-F_{L 21}$ (average effort $-\sigma$ ). The force $-F_{L 21}$ can be considered continuous over time along the direction $\mathrm{O}_{1}-\mathrm{O}_{2}$, and thus acts in a similar way to the Coulomb force $F_{C}$ (but it is $F_{C C}$ in fact) between two electric charges with the same sign.

\section{3) Action on SMPP1}

We now look at the situation with percussions $-p_{n}$ that act on the surface $S / M M$ of SMPP1 under the influence of SMPP2, and find that the position of the ,$-+u$ deformations in the ETH in the area of SMPP1 is completely symmetrical with regard to the situation of,$-+u$ arising from SMPP2, as described in 2) (Figure 5(a) and Figure 5(b)). Symmetry is also maintained in the case of the deformations,$+-u_{s}$. As a result, the normal/orthogonal $-p_{n}$ percussion forces exerted on SMPP1 will be symmetrical to those acting on SMPP2, and will result in a force $-F_{L 12}$ (on single EC) (of $-\sigma$ nature):

$$
-F_{L 12} \cong-F_{L 21}
$$

This force $-F_{L 12}$ will also be a Coulomb-type force $F_{C}$ of repulsion of SMPP1 by SMPP2 (but $F_{C C}$ in fact). 


\subsection{Interaction between Two Negative Immovable SMPNs through FVLs}

\section{1) General conditions of SMPNs:}

In this case, Figure 5(a) must be adapted to reflect the new situation: here, we have SMPN1 and SMPN2 (rather than SMPP1 and SMPP2), and the angles of the internal vortices and external rotations $+\omega /+\Omega$ must be reversed to become $-\omega /-\Omega$, while the directions of the arrows representing the sizes of the deformations,$+-u$ will be inverted to give,$-+u$, as explained in Section 2.4.

\section{2) Action on SMPN2:}

Here, we analyse the situation of the percussion forces produced by SMPN1 at point $M_{2}$ on the surface/membrane $S / M M$ of SMPN2, facing SMPN1 (Figure 5(a)-adapted).

The vibratory linear/longitudinal deformations (here, radial) both those in the ETH,,$-+u$, with the first $-u$, and the rest,$+-u$, as well as,$-+u_{s}$ at the $S / M M$ surface of the SMPN2 particle, are represented in the inset to Figure 5 (c) by a periodic zigzag line (ZL) with period $T$, depending on $t$.

Hence, the order of occurrence of the phases of the deformations,$+-u$ will be reversed twice: once due to the reversal of the direction of the $+u$ axis, and once from reporting to SMPN2, as described in Section 3.1. These then become the first positive,$+-u$ deformations in ETH around SMPN1, and the deformations at the surface $S / M M$ of SMPN2 will become the first negative ones,$-+u_{s}$ (Figure $5(\mathrm{c}))$.

At point $M_{2}$ on SMPN2 facing SMPN1 (Figure 5(a)-adapted, Figure 5(c)), we find that at time $t_{0}=0$, the deformations in the ETH will be positive deformations,$+-u$ in the ECs of the adjacent ETH, originating in SMPN1. In the case of the PC cells at the surface $S / M M$ of SMPN2 (Figure 5(c)), their first deformations are negative,$-+u_{s}$ and these appear in the opposite direction to the deformations,$+-u$, in the ETH, but with the same phase.

The peak points $\mathrm{P}_{\mathrm{i}}$ of the ZL curve of the periodic deformations,$+-u$ in the ETH coming from SMPN1 at various timeframes $T$, will be directly opposite to the peak points $\mathrm{P}_{\mathrm{i}}$ of the ZL curve for the,$-+u_{s}$ deformations in SMPN2. The compressive percussion forces $-p_{n}$ from ETH are focused in these contact points $\mathrm{P}_{\mathrm{i}}$ where the percussion forces $-p_{n}$ from SMPP2 are focused, (as part of PHA active phase from Section 2.3.), having opposed senses, so giving repulsion forces (Figure 5(c)).

In this case, normal/linear compression percussion forces $-p_{n}$ (negative compression effort $-\sigma$ ) will occur at point $\mathrm{M}_{2}$ on the near half of the surface $S / M M$ of SMPN2, over the area $S_{s}$ of a single EC (circle radius $r_{00}$ ), i.e. repulsion of SMPN2 from SMPN1. Hence, at the elementary surface $S_{s}$ corresponding to the central string $\mathrm{ST}_{0}$ of $\mathrm{ECs}$ at point $\mathrm{M}_{2}$, a continuous rejection force $-p_{n}$ will appear as an average over a period $T$ of periodic percussion forces $-p_{n}$. This force $-p_{n}$ will be continuous over time, repelling SMPN2 due to SMPN1, and is denoted as $-F_{L 21}$; it is created by periodic percussion forces $-p_{n p}$ through their av- 
erage effort - $\sigma$ over the elementary surface $S_{s}$ of an EC.

This repulsion force $-F_{L 21}$ (on a single EC) will be created by $p_{n}$ percussions through their average $-\sigma$ effort on the elementary $S_{s}$ surface of the EC, according to Figure 5(a)-adapted, Equation (6) being valid here also.

$$
-F_{L 21}=-p_{n}=S_{s}(-\sigma)
$$

Note 3.1, Note 3.2, Note 3.3 adjusted, and the adjusted Summary 3.1 from Section 3.1, final of point 2) are also valid here.

3) Action on SMPN1:

If we look at the case of SMPN1 under the influence of SMPN2, we find that the position of the deformations,$+-u$ within the area of SMPN1 are completely symmetrical to the those of,$+-u$ in the area of SMPN2, as explained in point 2) above (Figure 5(a)-adapted, Figure 5(c)). Symmetry is also maintained in the case of deformations,$-+u_{s}$

As a result, the percussion forces $-p_{n}$ (of $-\sigma$ nature) exerted on SMPN1 will be symmetrical to those acting on SMPN2. A resulting repulsion force $-F_{L 12}$ will also be exerted on SMPN1; this will also be a Coulomb-type force $F_{O}$ (but it is $F_{C C}$ in fact) which constitutes a repulsive force on SMPN1 by SMPN2. Equation (9a) is also valid here:

$$
-F_{L 12} \cong-F_{L 21}
$$

\subsection{Interaction between Immovable SMPP and SMPN through FVLs}

\section{1) General conditions of SMPP/SMPN:}

Figure 5(a) must be adapted to reflect this situation, in which SMPP1 is preserved and SMPP2 is replaced by SMPN2 (Figure 5(a)-adapted). Here, the angles of the internal vortices and external rotations $+\omega /+\Omega$ for SMPP1 must be reversed to become $-\omega /-\Omega$ for SMPN2, and the directions of the arrows representing the sizes of the deformations,$+-u$ will be adapted to each SMP, as described in Section 2.4.

2) Action on SMPN2: We will analyse the case of the forces created at point $M_{2}$ on the surface/membrane $S / M M$ of SMPN2 (Figure 5(a)-adapted). The linear/longitudinal vibratory deformations (radial in this case), both those in the $\mathrm{ETH},+,-u$ (in which the first has size $+u$, and the rest,$-+u$ ) and those at the surface of particle SMPN2,,$-+u_{s}$ (in which the first has size $-u_{s}$, and the rest ,$+-u_{s}$ ) are represented in the inset of Figure $5(\mathrm{~d})$ by a periodic zigzag line with period $T$, as function of $t$.

The order of the phases of the particle deformations will therefore be reversed at SMPN2, becoming,$-+u_{s}$ at the surface $S / M M$ (Figure 5(d)). At point $M_{2}$ (Figure 5(a)-adapted, Figure 5(d)) we find that at time $t_{0}=0$, the deformations of the ECs in the adjacent ETH will be with the first negative,,$-+u$, after reversing,$+-u$ directions of the deformations coming from SMPP1. And the longitudinal/radial deformations of PCs cells from the surface $S / M M$ of SMPN2 will be with the first,$-+u_{s}$ negative and the rest,,$+-u_{s}$. They will have the same direc- 
tion as the longitudinal/radial deformations,$-+u$ in ETH and will have the same phase, according to the HVFA hypothesis presented in Sections 2.3.1, 2.4.

Hence, at point $M_{2}$, the peak points $\mathrm{P}_{\mathrm{i}}$ (at which the percussion forces,$-+p_{n}$ are focused) of the broken line ZL of deformations,$-+u$ in the ETH coming from SMPP1 will occur at various timeframes $T$ in positions identical to the $P_{i}$ peaks in the ZL of the deformations,$-+u_{s}$ of SMPN2, thus twisting/penetrating themselves, having identical senses, so giving attraction forces (Figure 5(d)).

In this case, at the peak points $\mathrm{P}_{\mathrm{i}}$ in the ETH/SMPN2 surface $S / M M$ where the percussion forces $-p_{n}$ are focused, (as part of inversed PHA active phase from Section 2.3.1), positive percussion forces $+p_{n}$ (i.e. tensile forces) will be created in the ETH with regard to SMPN2. When applied to the surface of SMPN2, these positive percussion forces $+p_{n}$ will create an attraction force $+F_{L 21}$ on SMPN2, as shown in Figure 5(d).

On the elementary surface $S_{s}$ (circle with $r_{00}$ ) of an EC belonging to the central string $\mathrm{ST}_{0}$ of ECs, at point $M_{2}$, an average attractive percussion force $+p_{n}$ (over $\left.+p_{n}\right)$ will appear as an average over a time period $T$ of the percussions,$+-p_{n}$.

This average force will be continuous over time, due to the attraction of SMPN2 by SMPN1, denoted here as $+F_{L 21}$. This is created by percussion forces $+p_{n}$, by their average effort $+\sigma$ on the elementary surface $S_{\mathrm{s}}$ of an EC:

$$
+F_{L 21}=+p_{n}=S_{s}(+\sigma)
$$

This force $+F_{L 21}$ (on a single EC) corresponds to the $\mathrm{O}_{1}-\mathrm{O}_{2}$ direction of the connection $r$ between SMPP1 and SMPN2 (Figure 5(a)-adapted).

In Figure 5(a)-adapted, it is obvious that $+F_{L 21}$ is a mechanical force of attraction arising from periodic percussion forces $+p_{n}$ and from their average tensile stresses $+\sigma$, based on the mechanism described above and illustrated in Figure $5(\mathrm{~d})$; these forces vary as $1 / r^{2}$ over short distances, in the same way as $F_{C}$ :

$$
+F_{L 21}=f\left(r_{0}, r_{00}, p_{n}, \frac{1}{r^{2}}\right)
$$

The full form of function $f$ from Equation (11) can be determined in a similar way to that in Equation (8).

Here, we assume that,$-+u$ of the VFLs in the ETH within the area of SMPN2 have the same phase and same direction/sign as,$-+u_{s}$ from SMPN2; as a result, these percussion forces,$+-p_{n}$ (on a single EC) will give tensile stresses $+\sigma$, and periodic forces $+F_{L 21}$ of attraction to SMPN2 with period $T$. The force $+F_{L 21}$ is permanent over time in the direction of SMPN2; this force of attraction therefore acts in a similar way to the Coulomb force $F_{C}$ (but it is $F_{C C}$ in fact) between two electrical charges $+q /-q$ with opposite signs, although it is different in nature.

Here is also the case of the Note 3.1-adapted, of Note 3.3-adapted and of Summary 3.1-adapted from Section 3.1., final of point 2).

Note 3.2-adapted: This correlation between the periodic deformations,$-+u$ and,$-+u_{s}$ of SMPP2 and SMPP1 giving attraction force, is also ensured according to the mechanism described in Section 2.3.1, where the percussion forces $-p_{n}$ 
are focused, as part of superposition of a PHA active phase and of a PHR reactive phase.

3) Action on SMPP1: If we analyse the case of SMPP1 under the influence of SMPN2, we find that the position of the deformations,$-+u$ in the area of SMPP1 is similar to the case of,$-+u$ in the area of SMPN2, as described in point 2) above (Figure 5(a)-adapted, Figure 5(d)). Symmetry is also maintained in the case of deformations,$-+u_{s}$.

As a result, percussion forces $+p_{n}$ (of nature $+\sigma$ ) exerted on SMPP1 will be symmetrical with those exerted on SMPN2. The attraction force $+F_{L 12}$ (on a single EC) will also then appear on SMPP1, as attraction from the part of SMPN2.

$$
+F_{L 12}=+p_{n}=S_{s}(+\sigma)
$$

It should be noted that the force $+F_{L 12}$ also corresponds to the Coulomb attraction force $F_{C}$ between a positive and a negative charge. Equation (7) obviously applies here, in the same way as in Section. 3.1:

$$
F_{L 12} \cong F_{L 21}
$$

\section{Application of HFVI to the Interaction between Immovable SMPs in Ether via FVTs}

\subsection{Interaction of Two Immovable SMPPs in the Ether via FVTs}

1) General conditions of SMPPs: We consider here in HFVI, how the FVTs in the ETH are produced by the global rotations of SMPPs with rotation speed $+\Omega$, according to the mechanism basically described in Sections 2.3.1, 2.4 and 2.6.

Figure 6(a) shows a plane section perpendicular to the central axes of rotation, $\mathrm{O}_{1} Z$ and $\mathrm{O}_{2} Z$, of SMPP1 and SMPP2, which are parallel to each other, and perpendicular to the plane of Figure $6(\mathrm{a})$. The reference frame $\mathrm{O}_{1} x y z$ is attached to SMPP1, which is immovable in the ETH, with distance $D$ between them.

In the case of SMPPs we will admit through HVFI, that the deformations,$+-V_{s}$ of the $S / M M$ surface, are the result of the superposition of the effects of vibratory deformations $u_{s}$, with the general rotational movement with $+\Omega$ of the SMPP around the $\mathrm{O} z$ axis, according to the mechanism in Section 2.3., 2.4.

These deformations,$+-v_{s}$ are in fact equivalent/special deformations, which would create in the ETH the transversal vibrations/deformations,$+-v$, with $v_{s}=$ $V$, according to Section 2.6. These deformations,$+-V_{s}$ develops in two phases: active phase (PHA), when SMP acts upon ETH transmitting energy (only which counts), and passive phase (PHP), when ETH acts upon SMP, retransmitting energy.

The deformations,$+-v$ in the ETH are initially, cylindrically propagated, since they have cylindrical symmetry around $\mathrm{Oz}$ axis of rotation, but at great distances, they are spherical propagated.

In the case of SMPP, we will assume that the initial deformation $v_{s}$ are the result of a clockwise rotation of $S / M M$ with $+V_{s}$ (corresponding to the sense $+\Omega$, 


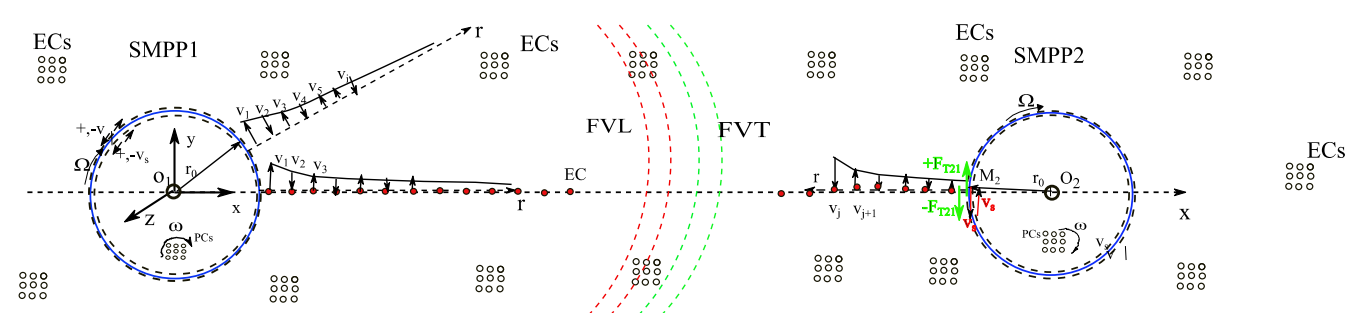

a). Situation of SMPP1/SMPP2 with deformation $v, v_{s}$ and tangential forces $F_{L 21}$

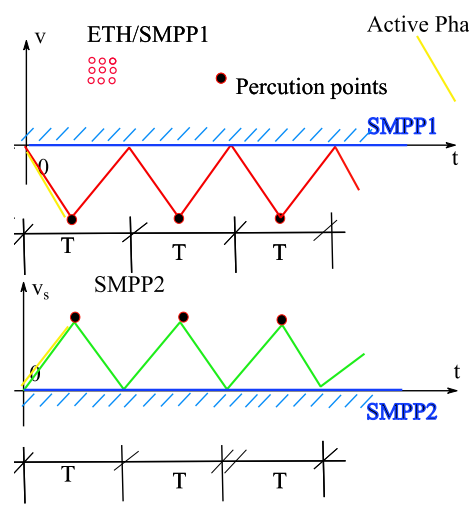

b). Grafic of v deformations in time and percution points upon SMPP2 from SMPP1 as counterclockwise first $\mathrm{F}$ force

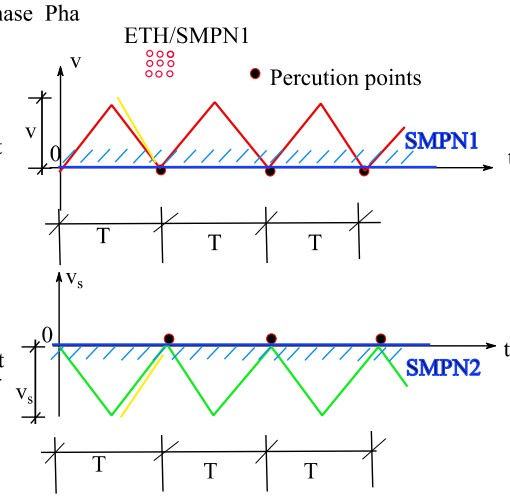

c). Grafic of v deformations in time and percution points upon SMPN2 from SMPN1

as counterclockwise first $\mathrm{F}$ force

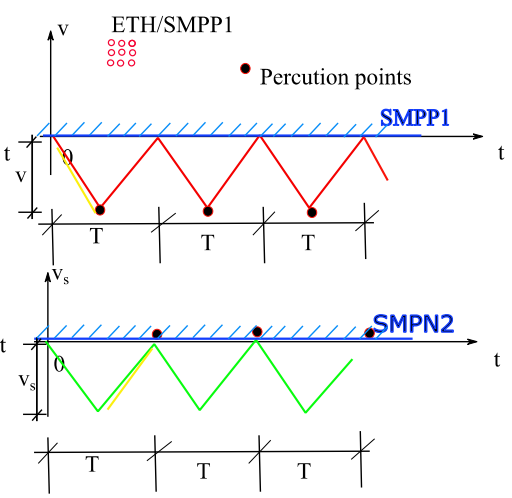

d). Grafic of v deformations in time and percutions points upon SMPN2 from SMPP1 as clockwise first $\mathrm{F}$ force

Figure 6. Physical mechanism of interaction between pairs of SMPs through their FVT vibrations in the ether, via percussions $p_{p}$, through the evolution in time of attached deformations $v$.

in PHA), that is denoted as positive here, followed by a counterclockwise rotation $-V_{\mathcal{s}}$, in PHR and then followed by pairs of oscillations,$+-V_{\mathcal{s}}$, and so on, with frequency $f_{t}$ and period $T_{t}$.

The deformation $+v_{s}$ corresponds to the rotation of the radius $r_{0}$ (of the point $\mathrm{M}$ situated on the parallel circle of the sphere of SMPP), clockwise around the $\mathrm{O} z$ axis through an angle,$+-\delta$ of vibrations, with size appropriate to the displacement,$+-V_{s}$. And the point $\mathrm{M}$ has the position angle $\lambda$, of the parallel circle (Figure 2 and Figure 6(a)).

Deformations from,$+-V_{s}$, will occur tangentially to the surface $S / M M$, as the alternating/vibratory displacements,$+-V_{s}$ of the $M$ point on the $S / M M$ surface (Figure 6(a)). The displacements $+v_{s}$ will correspond to the specific angular deformations $\gamma_{r}=v_{s} / r_{0}$, with frequency $v_{t}$.

For the SMPP, the initial displacement is considered to be $+v_{s}$, in PHA while for the SMPN the initial displacement will be $-v_{s}$, in PHR corresponding to the two types of effects from the quasielectrical charges $+q,-q$, of SMPP and SMPN respectively, as defined in Section 2.5.

Once the deformations,$+-V_{s}$ appear, tangential percussion forces,$+-p_{t}$ will arise at the surface of the SMP upon ETH, through the MM membrane at the surface $S$ of the SMP. These periodic percussion forces,$+-p_{t}$ (on a single EC) 
will then be exerted on ECs in the adjacent external ETH, and thus deformations ,$+-V$ are produced in ETH. And being associated to deformations,$+-V$, the fundamental vibrations FVTs will also arise in ETH (Figure 2, Figure 6(a)).

This mode of occurrence of FVT, when the PCs cells or GPCs/quarks inside the SMP may continuously rotate individually with rotation speed,$+-\omega$, allowing concomitantly the general rotation of the SMP with rotation speed $+/-\Omega$ of the membrane MM on the surface $S / M M$ of the SMP.

This model of FVT, is well correlated with other observed physical phenomena, such as the storage of energy $E_{0}$ in a GPC, with rotation speed $\omega$, and with the spin of SMPs, due to SMP general rotation with speed $+/-\Omega$ (Figure 2, Figure 6(a)).

Thus, the spin arises from the effect of the rotations $\Omega$ of PCs at the outer surface of the SMP, since the rotations $+/-\omega$ of the inner PCs/quarks will be cancelled in pairs at the tangent points of the PCs, where the directions of the rotations $+/-\omega$ are opposite.

This is similar to the occurrence of Ampere currents in magnetism, although the energy $E$ is present in the rotations $+/-\omega$ of all inner PCs/quarks, and will be accumulated and preserved.

Figure 6(a) indicates the direction of the transversal vibrational deformations ,$+-V$ of the ECs in ETH, at circumferences of different rays $r_{p}$ related to the FVTs of SMPP1's with period $T_{T}$ The transversal deformations,$+-V_{s}$ of the surfaces $S / M M$ of the SMPs are also shown.

These circular, pulsating deformations,$+-V$ of the ETH are imprinted by circular pulsating,$+-V_{s}$ deformations in the surface $S / M M$ of SMPP1 in PHA, in the first layer of the adjacent EC cells in ETH, as described in Section 2.3.1, 2.4, 2.6.

These vibrational deformations,$+-V$ are then transmitted/imprinted in a step-by-step way to all the external ECs layers within the ETH through the mechanism of periodic transversal percussion forces,$+-p_{t}$ which produce FVTs, starting from SMPP1. The values of these deformations,$+-V_{i}$ will decrease as $v_{0}>v_{1}>v_{2}>v_{3}>\cdots>v_{n}$, (Figure 6(a)) corresponding to a variation of $1 / r^{2}$, in a similar way to Coulomb's law, as described in [1] [8].

The deformations,$+-V$ will create in ETH, associated specific angular deformations or strains,$+-\gamma$, and this is possible in the case of HM16 crystalline variant A.

We assume that the FVTs of an SMP start from $S / M M$ as a set of cylinders around the $\mathrm{O} z$ axis of rotation, which then expand to the whole ETH, becoming spherical at large distances.

We must consider that the,$+-v$ deformations, are local, discrete, on a single EC, as a quantum effect.

\section{2) Action on SMPP2:}

We will first analyse the action of SMPP1 on SMPP2 via FVTs under the general conditions of the behaviour of SMPs in HFVI, as described in Sections 2.3.1, 


\section{4., 2.6. and above.}

Here, we examine the case where the $\mathrm{Oz}$ axes of both SMPs are parallel, to simplify the analysis. We analyse a direct interaction between two SMPs, given by percussions,$+-p_{t}$ that are associated with vibrational deformations,$+-v$ via a single string $\mathrm{ST}_{0}$ of ECs, and which is central, linear and direct between $\mathrm{O}_{1}$ and $\mathrm{O}_{2}$ (Figure 6(a)).

The total forces, including the indirect interaction forces between two SMPs via multiple STs as series of curved paths $I_{i}$ and their effect through,$+-v$ of the FVTs upon SMPP2, can be analysed separately in the same way as for the vibrations,$+-u$ of FVLs; these act as a corrected Coulomb force $F_{\mathrm{CC}}$ as described in [2] [8], although here these,$-+v$ originate in the FVTs.

Figure $6(\mathrm{~b})$ shows how the first deformations $+v$ of the ECs in the adjacent ETH, derived from SMPP1, become $-V$ (due to the reversal of the direction of the axis $+v$ ) when applied to SMPP2.

In this simple case, at point $M_{2}$ on SMPP2, a series of tangential percussion forces,$+-p_{t}$ during PHR will be transmitted from SMPP1 via the ETH, with the first being the negative $-p_{t}$ due to a reversal of the sense of $+v$ when switching from SMPP1 to SMPP2 (Figure 6(a), Figure 6(b)).

At point $M_{2}$, we therefore find that at time $t_{0}=0$, the first deformations of the ECs will be $-V$, during PHA of SMPP1 i.e. in the opposite direction to the first deformations $+v_{s}$ in the PCs within SMPP2, although these deformations have the same phase, and during PHA of SMPs.

The peaks in the zigzag line $\mathrm{ZL}$ at the points $\mathrm{P}_{\mathrm{i}}$ (where the percussion forces ,$-+p_{t}$ are focused, as effects of PHA, Figure 6(b)), i.e. periodic deformations ,$-+v$ of the ETH at various timeframes $T / 2$, will therefore be directly opposite to the direction of movement of the peaks $\mathrm{P}_{\mathrm{i}}$ of the deformations,$+-v_{s}$ in SMPP2 having opposed senses, so giving repulsion forces. (Figure $6(\mathrm{~b})$ ). It follows that the percussions,$-+p_{t}$ will constitute forces that oppose the direction of the deformations,$+-V_{s}$ in the surface of SMPP2, and are thus also opposite to the corresponding forces,$+-p_{s t}$ afferent to the SMPP2 surface $S / M M$, as effects of PHA superposition from Section 3.2.1, indicating rejection of SMPP2 from SMPP1, which finally will be reciprocal.

In this way, at point $\mathrm{M}_{2}$ on the elementary surface $S_{s}$ of a single EC (a circle with radius $r_{00}$ ) of the central string $\mathrm{ST}_{0}$ of ECs, tangential periodic percussions ,$-+p_{t}$ with period $T / 2$ will arise.

The percussion forces,$+-p_{t}$ from ETH (Figure 6(b)), will be opposed to the deformations,$+-v_{s}$ in SMPN2, and hence form tangential efforts,$-+\tau$ restraining the deformations in SMPN2 and mitigating its rotation, giving:

$$
-,+\tau=-,+p_{t} / S_{s}
$$

This will result in an average percussion force,$-+p_{t}$ (over,$-+p_{t}$ and thus ,$-+p_{t}=-,+p_{t} / 2$ ), as an average force over a semi-period $T / 2$, noted with,$-+F_{T 21}$, derived from percussion forces,$-+p_{t}$ (on a single EC) via the average periodic effort,$-+\tau$ and this will have the form: 


$$
-,+F_{T 21}=-,+p_{t}=S_{s}(-,+\tau)
$$

The force,$-+F_{T 21}$ will therefore be a periodic tangential force that produces a alternating rotation in SMPP2 by SMPP1, while creating a braking/attenuating effect on the periodic vibration,$+-v_{s t}$ or on rotation,$+-\Omega$, of SMPP2, as shown in Figure 6(a).

This tangential force,$+-F_{T 21}$ will be continuous over $T / 2$, due to SMPP1, and is created by the percussion forces,$-+p_{t p}$, through their average,$-+\tau$ effort on the elementary surface $S_{s}$ of an single EC.

This force,$-+F_{T 21}$ corresponds to the direction/line $\mathrm{O}_{1}-\mathrm{O}_{2}$ of direct connection $I_{0}$ through $\mathrm{ST}_{0}$ between SMPP1 and SMPP2 (Figure 6(a)).

From Figure 6(a), it can be seen that the force,$-+F_{T 21}$ also corresponds to a special tangential force of Coulomb type $F_{C}$ between two positive charges $+q /+q$, although with a different nature. The nature of,$-+F_{T 21}$ is obvious; it is a tangential mechanical force derived from the tangential percussion forces,$+-p_{p}$ and from their average efforts,$-+\tau$, according to the mechanism described above and illustrated in Figure 6(b). This force varies as $1 / r^{2}$ over short distances, in the same way as $F_{C}$ :

$$
F_{T 21}=f\left(r_{0}, r_{00}, p_{t}, \frac{1}{r^{2}}\right)
$$

The full form of the function $f$ in Equation (16) can be established on an experimental basis depending on the geometry of the SMPs (radius $r_{0}$ ) and on the ECs (radius $r_{00}$ ), and also on the positions of the axes $\mathrm{Oz}$.

As a result, from the percussions,$-+p_{t}$ arising from SMPP1, and hence also from the force,$-+F_{T 21}$, acting tangentially on SMPP2 (with radius $r_{0}$ ), a moment ,$-+M_{T 21}$ of rotation (periodic in the same way as,$-+V$ ) will result:

$$
-,+M_{T 21}=-,+F_{T 21} r_{0}
$$

Since the deformations,$-+V$ at the extreme points $\mathrm{P}_{\mathrm{i}}$ on the lines of variation ZL over time $t$ for ETH and near SMPP2 have signs/directions that are opposite to those of the deformations,$+-V_{s}$ (Figure $6(\mathrm{a})$ and Figure $6(\mathrm{~b})$ ), then the percussions,$-+p_{t}$ (including the forces,$-+F_{T 21}$ and their efforts,$-+\tau$ ) will be opposed to,$+-V_{s}$, so representing a decrease in the,$+-\gamma_{0}$ rotational trend of SMPP2 due to SMPP1.

As a result, the moment,$-+M_{21}$ will also be a periodic/vibrational rotation moment on SMPP2 with a braking effect on its oscillations/rotations due to the influence of SMPP1, which is unknown at present and may have a quantum interpretation.

Note 4.1: This correlation in the periodic deformations,$-+V_{s}$ and,$-+v$ between SMPP2 and SMPP1 giving repulsion forces,$-+F_{T 21}$ also arises as described in Section 1 for SMPAC and HCO, through the suitable commands for reversing the direction,$-+v$ provided by the NCs of SMPP1 and SMPP2.

Note 4.2: This correlation between the periodic deformations,$-+V_{s}$ and,$-+V$ of SMPP2 and SMPP1 giving repulsion forces,$-+F_{T 21}$, is also ensured according 
to the mechanism described in Section 2.3.1., where the percussion forces $-p_{t}$ are focused, as part of superposition of two PHA active phases, having opposed senses, so giving repulsion force,$-+F_{T 21}$ and braking moment $M_{T 21}$.

Note 4.3: We admit in HFVI that the above forces action is at the single EC area of the,$+-p_{t}$ and the equivalent force,$+-F_{T 21}$ in Equation (16) and also the moment ,$-+M_{T 21}$ in Equation (17). So,$+-F_{T 21}$ and,$-+M_{T 21}$ represents the forces/moments with minimum intrinsic/predetermined sizes for the SMPs and the ETH, which constitute the minimum forces/moments $\Delta F / \Delta M$ in quantum mechanics. Hence, these forces $F_{T 21}$ may constitute a kind of special magnetic field $Q H_{s}$ whose nature needs to be researched in future.

\section{3) Action on SMPP1:}

In the case of the action of the force from SMPP2 on SMPP1, due to the symmetry of the situation (Figure 6(a)), the same result as in point 2 ) will be obtained and the periodic,$+-F_{T 12}$ force will result :

$$
-,+F_{T 12}=-,+p_{t}=S_{s}(-,+\tau) \cong-,+F_{T 21}
$$

The periodic rotation moment,$-+M_{T 12}$ will also arise:

$$
-,+M_{T 12}=-,+F_{T 12} r_{0} \cong-,+M_{T 21}
$$

where,$-+M_{T 12}$ is a periodic/vibrational moment of rotation on SMPP1 with a braking effect on its oscillation due to SMPP2.

\subsection{Interaction of Two SMPNs that are Immovable in the Ether, through FVTs}

\section{1) General conditions of SMPNs:}

In this case, Figure 6(a) must be adapted to represent SMPN1 and SMPN2 with the internal rotations $\omega$ and external rotations $\Omega$ that are reversed, becoming $-\omega$ and $-\Omega$, respectively. The senses of the arrows representing the deformations,$+-v$ will also be inverted (Figure $6(\mathrm{a})$ ).

Thus, the order of occurrence of the phases of the deformations,$+-v$ will be reversed on both sides, becoming,$-+v$, in the ETH around the SMPN and,$-+V_{s}$ (with the first of size $-v_{s}$, and the rest of size,$+-v_{s}$ ) at the surface $S / M M$ of the SMPN.

In the case of interaction between two negative SMPs, SMPN1 and SMPN2, by means of FVTs, the situation is similar to that discussed in Section 4.1 above, with Figure 6(a)-adapted, showing the changed directions of $\omega, \Omega$ angles and of ,$+-v$ senses.

This is because the difference between an SMPN and an SMPP in respect of FVTs consists only of the senses of transversal deformation,$+-v$ and the related ,$+-\gamma$ angle, as illustrated in HFVI in Section 2. This means that for an SMPN, the initial rotation is $-v$, and $-\gamma$, i.e. counterclockwise. This reversed direction of the initial deformation/vibration has no influence on the size of the forces $F_{T}$ or the moment $M_{T}$, but only on their directions in Equation (18), Equation (19).

\section{2) Action on SMPN2:}


The transversal/pulsating deformation,$-+V_{s}$ (initially with size $-v_{s}$, and then ,$\left.+-V_{s}\right)$ at point $M_{2}$ on the surface $S / M M$ of SMPN2, and deformations/vibrations ,$+-v$ in the adjacent ETH, is represented in the inset in Figure 6(c), and depends on the time $t$ over a period $T$. This results in a periodic zigzag line $\mathrm{ZL}$, due to the pulsating and periodic character of,$+-V$ and,$+-V_{s}$ with time step $T / 2$.

The first deformations $-v$ of the ECs in the ETH adjacent to SMPP1 become $+v$ in Figure $6(\mathrm{c})$ near SMPN2 (due to the change in direction of the axis $+v$ ).

At the central point $M_{2}$ on the face of SMPN2 facing SMPN1 (Figure 6(a)-adapted, Figure $6(\mathrm{c})$ ), we find that at time $t_{0}=0$, the deformations,$+-v$ in the ECs caused by SMPN1 in the adjacent ETH are opposite in direction/sense to the deformations,$-+\mathrm{v}_{s}$ of the PCs of MM within SMPN2, although they have the same phase.

Hence, the peaks at points $P_{i}$ where the percussion forces,$+-p_{t}$ are focused (as part of PHA active phases superposition, from Section 2.3.1.), having opposed senses, so giving repulsion forces (Figure $6(\mathrm{c})$ ). So on the line ZL of periodic deformations,$+-v$ in the ETH at various timeframes $T / 2$, deformations,$+-v$ will be directly opposite to the peaks of the deformations,$-+v_{s}$ in SMPN2 with period $T / 2$ indicating rejection of SMPN2 which finally will be reciprocal (Figure $6(\mathrm{c}))$.

The transversal vibratory deformations,$+-v$ of the ECs in the ETH adjacent to SMPN2 will be accompanied by periodic transversal/tangential percussion forces,$+-p_{t}$ at point $M_{2}$ (initially positive, on a single EC) on the nearby half-surface $S / M M$, of the nature of some tangential,$+-\tau$ efforts, and these are also vibrational. The average of these efforts,$+-\tau$ at point $M_{2}$ on the area $S_{s}$ of an single EC projection (with radius $r_{00}$ ) on the half-surface $S$ close to SMPN2, will give the force,$+-F_{\mathrm{T} 21}$. The first impulse of,$+-F_{T 21}$ is counterclockwise, starting from SMPN1, and this is reversed to the clockwise sense on SMPN2, as explained in Section 2 (Figure 6(a)-adapted, Figure 6(c)).

In this way, an average percussion force,$+-p_{t}$ (over,$+-p_{t}$, thus,$+-p_{t}=$ ,$\left.+-p_{t} / 2\right)$ will appear at point $\mathrm{M}_{2}$ as an average force over a half-period $T / 2$, denoted as,$+-F_{T 21}$ (on a single EC), arising from percussion forces,$+-p_{t}$ through their average periodic effort,$+-\tau$, and will have the form:

$$
+,-F_{T 21}=+,-p_{t}=S_{s}(+,-\tau)
$$

Thus, the force,$+-F_{T 21}$ (on a single EC) will be a periodic tangential force on $S / M M$ that produces a rotation in SMPN2 due to SMPN1, but creates a braking/attenuating effect on its own periodic rotations,$-+v_{s}$ as shown in Figure 6(a)-adapted.

This tangential force,$+-F_{T 21}$ corresponds to the direction/line $\mathrm{O}_{1}-\mathrm{O}_{2}$ of direct connection $l_{0}$ through $\mathrm{ST}_{0}$, between SMPN1 and SMPN2 (Figure 6(a)-adapted).

As a result of the percussion forces,$+-p_{t}$ and hence the forces,$+-F_{T 21}$ (on a single EC) acting tangentially on SMPN2 (radius $r_{0}$ ), a rotation moment,$+-M_{T 21}$ (periodic in the same way as,$+-v$ ) will emerge:

$$
+,-M_{T 21}=+,-F_{T 21} r_{0}
$$


As explained in Sec. 4.1, also here, since the deformations,$-+v$ at the extreme points of the lines ZL of variation during $t$ for ETH and for SMPN2, have signs/senses that are opposite to the deformations,$+-v_{s}$ (Figure 6 (a)-adapted, Figure $6(\mathrm{c})$ ), the percussions,$-+p_{t}$ (including the forces,$-+F_{T 21}$ and their efforts $-,+\tau)$ will be opposite to,$+-V_{s}$, representing an attenuation in the rotation of SMPN2,$+-\gamma_{0}$ due to SMPN1.

The moment,$+-M_{T 21}$ will therefore be an alternating rotation moment on SMPN2 due to the influence of SMPN1, which will tend to oppose the own moment,$-+M_{T 21}$ of SMPN2 by braking.

Here, Note 4.1.-adapted, Note 4.2.-adapted and Note 4.3.-adapted from Section 4.1 point 2 ) are also valid here.

\section{3) Action on SMPN1:}

In the case of the action of SMPN2 on SMPN1, due to the symmetry of the deformations $V$, and hence also of the percussion forces $p_{t}$ (Figure 6-adapted), the same result as in point 2 ) will be obtained for the force,$+-F_{T 12}$ and the moment,$+-M_{12}$ given in Equation (20) and Equation (21):

$$
\begin{gathered}
+,-F_{T 12}=+,-p_{t}=S_{s}(+,-\tau) \cong+,-F_{T 21} \\
+,-M_{T 12}=+,-F_{T 12} r_{0} \cong+,-M_{T 21}
\end{gathered}
$$

The moment,$+-M_{T 12}$ will be a periodic/oscillating rotational moment on SMPN1 with a braking effect on the own oscillation/rotation,$-+M_{s T 1}$ of SMPN1.

\subsection{Interaction between Immovable SMPP and SMPN, in the Ether, via FVTs}

\section{1) General conditions of SMPP/SMPN:}

In this situation, Figure 6(a) must be adapted to represent SMPP1 and SMPN2, with internal rotation $\omega$ and external rotation $\Omega$, which are reversed for SMPN2 to give $-\omega$ and $-\Omega$, respectively, and the senses of the arrows of the deformations ,$+-V$, will be inverted for SMPN2 (Figure 6(a)) as discussed in Section 2.

Thus, the order of occurrence of the phases of deformations,$+-V$ will be reversed, becoming,$-+v$, in the ETH around SMPN2 and remaining,$+-V_{s}$ (with initial size $+v_{\mathrm{s}}$ and then,$-+v_{\mathrm{s}}$ pairs) at the surface $S / M M$ of SMPP1.

\section{2) Action on SMPN2:}

The transversal/circular deformations,$-+V_{s}$, as vibrations at the point $M_{2}$ on the surface $S / M M$ of SMPN2, and the FVT deformations/vibrations,$-+v$ in the adjacent ETH, are represented in detail in Figure 6(d), and depend on time $t$, over periods $T$.

The first deformations $+v$ of the ECs in the ETH adjacent to SMPP1 become $-v$ in Figure 6(d) near SMPN2 (due to the change in direction of the $+v$ axis) when applied to SMPN2.

At the central point $M_{2}$ on the face of SMPN2, the zigzag lines ZL of the deformations,$-+v$ and,$-+V_{s}$ (Figure $6(\mathrm{~d})$ ) will result with the same sign (where the first deformation in ETH will be $-/+v$ ) with time step $T / 2$. These deformations have the same phase. 
The extreme peaks at points $\mathrm{P}_{\mathrm{i}}$ where the percussion forces $-p_{t}$ are focused as part of PHA active phases superposition, from Section 2.3.1., having identical senses, so giving attraction forces (Figure 6(d)). So on the ZL line of the periodic deformations,$-+V$ in the ETH at various timeframes $T / 2$, the deformations ,$-+v$, will therefore match the peaks in the deformations,$-+v_{s}$ in SMPN2, and since they have the same direction/senses, these lines will twist/penetrate themselves indicating attraction of SMPN2 which finally will be reciprocal attraction (Figure 6(d)).

The transversal vibratory deformations,$-+v$ of the ECs in the ETH adjacent to SMPN2 will be accompanied by periodic transversal/tangential percussion forces,$-+p_{t}$ at point $M_{2}$ (initially negative) at the $S / M M$ half-surface, of the nature of some tangential,$-+\tau$ efforts, which are also vibrational. The average of these efforts,$-+\tau$ at point $M_{2}$ on the area $S_{s}$ of an single EC projection (radius $r_{00}$ ), on the surface $S / M M$ close to SMPN2 will give the force,$-+F_{T 21}$ (where the initial impulse of $-F_{721}$ is clockwise, starting from SMPN1, but is reversed to counterclockwise for SMPN2) as discussed in Section 2.3.1. (Figure 6(a)-adapted, Figure 6(d)).

In this way, an average percussion force,$-+p_{t}$ (over,$-+p_{t}$ ) will appear at point $\mathrm{M}_{2}$ as an average force over time with period $T / 2$, denoted as,$-+F_{T 21}$, (on a single EC) arising from the percussion forces,$-+p_{t}$ through the average periodic effort,$-+\tau$, and will have the form:

$$
-,+F_{T 21}=-,+p_{t}=S_{s}(-,+\tau)
$$

Thus, the force,$-+F_{\mathrm{T} 21}$ will be a periodic tangential force that produces a oscillatory rotation in SMPN2 due to SMPN1, and creates a boosting effect for its own periodic rotation,$-+v_{s}$ or,$-+\Omega$, (Figure 6(a)-adapted, Figure $6(\mathrm{~d})$ ).

This tangential force,$-+F_{T 21}$ corresponds to the line $\mathrm{O}_{1}-\mathrm{O}_{2}$ of direct interaction line $I_{0}$ through $\mathrm{ST}_{0}$, between SMPN1 and SMPN2 (Figure 6(a)-adapted). Here, the force,$-+F_{T 21}$ is a Coulomb-type force but with a different nature, arising from VFTs.

As a result of the percussion forces,$-+p_{t}$ and hence the forces,$-+F_{T 21}$ due to SMPN1 tangentially acting on SMPN2 (radius $r_{0}$ ), a special rotational moment ,$-+M_{T 21}$ (periodic in the same way as,$-+v$ ) will emerge:

$$
-,+M_{T 21}=-,+F_{T 21} r_{0}
$$

The alternating rotation moment,$-+M_{T 21}$ (on a single EC) will act on SMPN2 due to the influence of SMPN1, tending to boost SMPN2's own moment ,$-+M_{s 21}$.

It can also be seen from Figure 6(a)-adapted that the,$-+F_{T 21}$ force also corresponds to a Coulomb force $F_{C}$ between two different charges $+q /-q$, although it has a different nature.

Note 4.1.-adapted, Note 4.2.-adapted and Note 4.3.-adapted, from Section 4.1 point 2 ) are also valid here.

\section{3) Action on SMPP1:}


In the case of the action of SMPN2 on SMPP1, due to the anti-symmetry of the situation (Figure 6(a)-adapted, Figure 6(d)), the result described in point 2) will also be obtained for the force,$-+F_{T 12}$ and the moment,$-+M_{T 12}$, as given in Equation (26a) and Equation (26b):

$$
\begin{gathered}
-,+F_{T 12}=-,+p_{t}=S_{s}(-,+\tau) \cong-,+F_{T 21} \\
-,+M_{T 12}=-,+F_{T 12} r_{0} \cong-,+M_{T 21}
\end{gathered}
$$

The resulting force,$-+F_{T 12}$ and the moment,$-+M_{T 12}$ on the elementary surface $S_{s}$ (on a single EC) will produce a boost in its own rotation,$-+V_{s}$ and its own moment,$-+M_{s 21}$.

\section{Effect Produced by FVs in ETH, Created by the Ordered Orientation of SMPs from an Immobile GSMP, Creating $Q E$ and $Q H$ Fields}

\subsection{FVL Vibrations Creating $Q E$}

We now consider the situation of a group of SMPs, GSMP1, that are homogeneous in electrical nature ( $\mathrm{P}$ or $\mathrm{N}$ ), and ordered in terms of their orientation on the $\mathrm{O} z$ axis (Figure 7). These SMPs will each transmit FVL waves in an AI-zone of the ETH near SMP2, in the presence of which vibrational deformations of type,$+-u$ and strains,$+-\varepsilon$ respectively will occur within the EC network of the ETH (Figure 7). These vibrations of the linear strains,$+-\varepsilon$ will be oriented in one direction and will have the same sense, and thus will accumulate from all the SMPs in the group within the AI. As a result, a sum of oscillatory percussions $\Sigma+,-p_{n}$ (on a single EC) will appear, which will act on SMP2 as explained in Section 3. Then, the sum of oscillatory forces $\Sigma+,-F_{L 21}$ from Equation (9a) will be developed, with attraction/repulsion effect on SMP2 depending on the type (P or $\mathrm{N})$ of the SMP2 and GSMP1 pair, as discussed in Section 3.

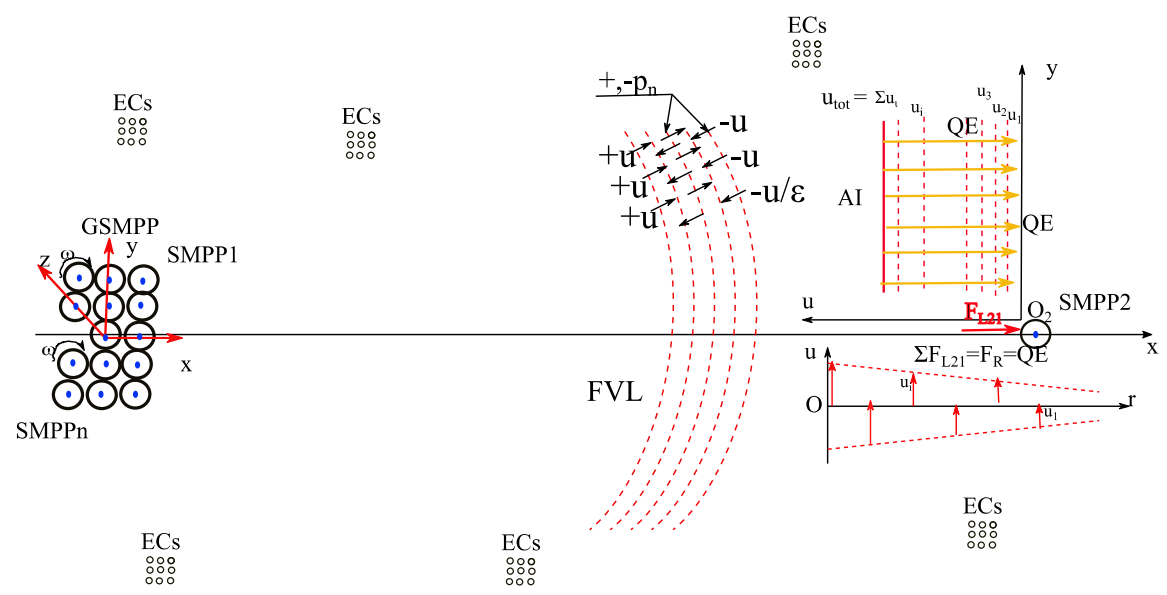
a). Zone of SMPP1
b) Zone of ETH free
c). Zone of SMPP2

Figure 7. Mechanism of interaction of percussion forces between immobile GSMP and a SMPP, via FVLs and deformations +,- $u$, creating a quasielectric field $Q E$ in the ETH. 
Thus, the effect of,$+-F_{L 21}$ from the GSMP1 group of ordered particles accumulates, giving a resulting force $F_{R}$ acting on SMP2 that is equivalent to the presence of a stronger quasielectric field $Q E$ (Figure 7).

Here, $Q E$ has a clear physical explanation, as it is produced by the normal percussion forces,$+-p_{n}$ (on a single EC, only in PHA) created by the FVLs from GSMP.

This $Q E$ field will produce the same physical effects as the $E$ field; the nature of $Q E$ is clear here, as it is an effect of the percussion forces $p_{i}$ created by the strains,$+-\varepsilon$ produced by the FVLs from the GSMP.

\subsection{FVT Vibrations Creating a Special $Q H_{s}$}

The FVTs of a stationary group of SMPs, GSMP1, can create in a certain AI near SMP2, from space/ETH, a special state of ordering of the vibrational deformations,$+-V$ and the associated angles,$+-\gamma$ from the EC network of the ETH (Figure 8).

This situation can only occur if GSMP1 has component SMPs that are homogeneous in nature ( $\mathrm{P}$ or $\mathrm{N}$ ) and are ordered in terms of their orientation and the directions of their $\mathrm{Oz}$ axes. Then the positions and orientations of the angles ,$+-\gamma$ in the AI zone of ETH produced by all SMPs, will have the same direction and sense, cumulating from all SMPs in the GSMP (Figure 8).

As a result, pulsating tangential percussion forces,$+-p_{t}$ will arise as a sum, $\Sigma+,-p_{t}$ (on a single EC) and will act tangentially on the surface $S / M M$ of SMP2 as described in Section 4. At the same time, the associated forces accumulate as $\Sigma+,-F_{T 21}=+,-F_{R T 21}$, and this resultant tangent force will create the vibrational/special moment of torsion,$+-M_{S R}$ (on a single EC) that acts on SMP2. The moment,$+-M_{S R}$ will have a damping or boosting effect on the rotations/vibrations of SMP2, based on the types of SMP2 and GSMP (P or N), as explained in Section 4.

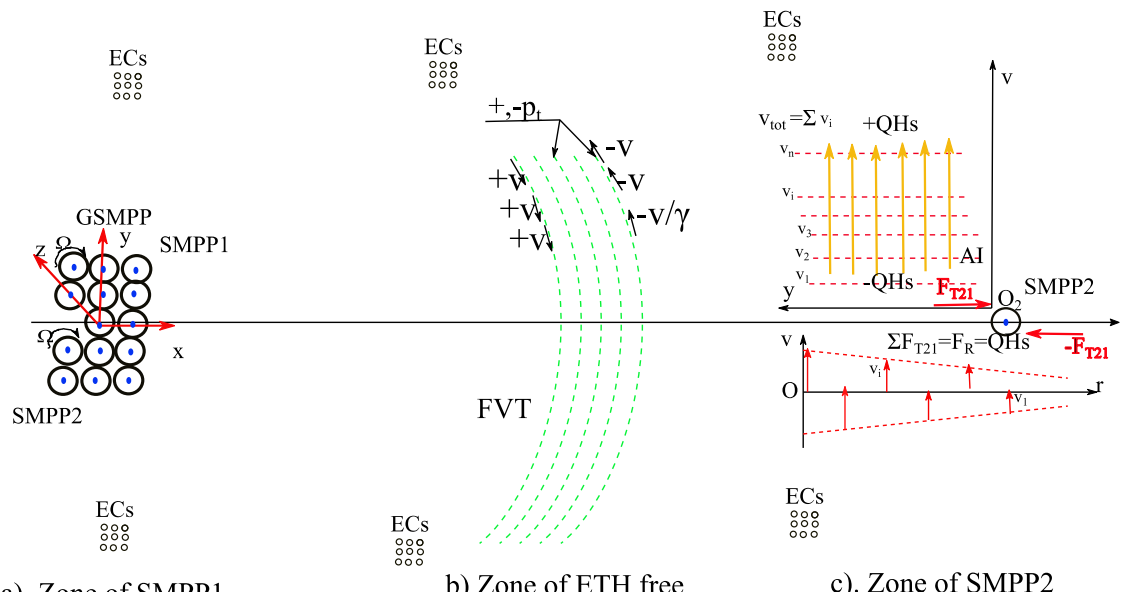

a). Zone of SMPP1

b) Zone of ETH free

c). Zone of SMPP2

Figure 8. Mechanism of interaction of percussion forces between an immobile GSMP and a SMPP, via FVTs and deformations,$+-v$, creating a quasimagnetic special field $Q H_{s}$ in the ETH. 
This damping or boosting action depends on the relative position of the axes $\mathrm{O} z$ of SMP2 and of GSMP; a parallel direction gives the best result, due to the highest interference of the associated strains $\gamma$.

The creation of a vibrational moment,$+-M_{S R}$ (on a single EC, only in PHA) acting on the surface $S / M M$ of an SMP2 is equivalent to the presence in the AI of a more powerful vibrating quasimagnetic special field,$+-Q H_{s}$; although this has no actual equivalent field in physics, it may explain some of the quantum properties of SMPs (Figure 6).

\section{Effect of FVs in the ETH Created by a Mobile SMP with Velocity $V$}

\subsection{FVLs of a Mobile SMP}

For a dynamic state of the SMP1/SMP2 pair, when SMP2 moves with velocity $V$ along the Ox axis through the ETH (Figure 9), an additional effect will appear, namely a dynamic component of the deformation,$+-u$ with size $\Delta u$, oriented in the $x$ direction.

This dynamic component $+\Delta u$ represents the displacement of SMP2 in the direction of $V$ for a half-period $T / 2$, during which the deformation,$+-u$ of the surface $S / M M$ of SMP2 occurs.

The deformation $+\Delta u$ in the area of SMP2 will then be transmitted in a radial/spherical way throughout the rest of ETH, and will reach SMP1, but with values $+\Delta u$ ' decreasing as $1 / r^{2}$ according to Coulomb's law (in the same way as,$+-u$, described in Section 3).

The new effect $+\Delta u$ or the associated $+\Delta \mathcal{E}$, will cause a change of $\Delta \mathcal{\varepsilon}$ in,$+-\varepsilon$, including in the AI of SMP1, and will therefore create a modification of the force ,$+-F_{L 12}$ on SMP1, as described in Section 3, but only when the direction of $V$ coincides with the direction $\mathrm{O}_{1}-\mathrm{O}_{2}$ (Figure 9).

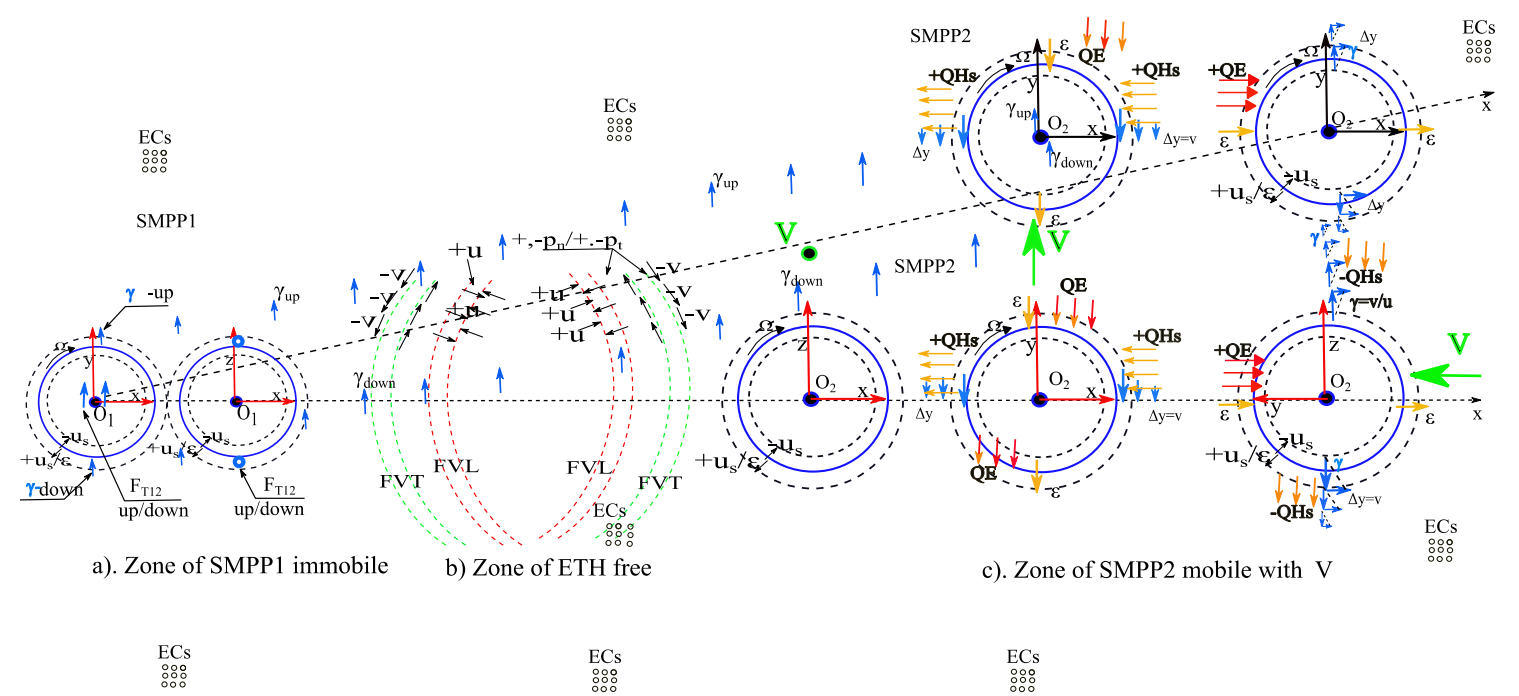

Figure 9. Deformations $u$ and $v$ in the interaction between mobile SMP2 and the EYH resulting quasielectric $(Q E)$ and quasimagnetic $(Q H)$ fields in the ETH. 
We also note that the deformation $+\Delta u$ is discrete and vibrating, due to the vibrating nature of,$+-u$ with which it is associated, and this vibration will be transmitted to $+\Delta \varepsilon$ and $+\Delta F_{L 12}$.

The new effect $+\Delta u$ will produce a change in the base force $+F_{L 12}$, (on a single EC, only in PHA) as explained in Section 3, by a new force $+\Delta F_{L 12}$ of repulsion/attraction type, of SMP1 by SMP2 due to velocity $V$.

From the reciprocity of the interaction discussed in Section 3, a similar modification of the force $+\Delta F_{L 21}$ on SMP2 due to SMP1 will be created, with size $+\Delta F_{L 21}=+\Delta F_{L 12}$. This force $+\Delta F_{L 21}$ is the reaction of the ETH acting on SMP2, opposing to its movement with speed $V$.

We note here that $\Delta x$ is the displacement of SMP2 during the period $\Delta t$ of the expansion of SMP2, where $2 u / 2=u$, during a semiperiod $T / 2$. We can therefore write:

$$
\Delta x=\Delta t \cdot V
$$

As a result, the total deformation $u_{t}$ of the point $M_{2}$ located on the surface $S / M M$ of SMP2 within a semi-period $T / 2=\Delta t$ will be:

$$
u_{t}=u+\Delta x=u+\Delta t \cdot V
$$

The time $\Delta t$ over which the deformation $u$ takes place, with its own velocity of deformation $c_{u}$ of the surface $S / M M$, during a single vibration $T / 2$ within the FVL, will be:

$$
\Delta t=\frac{u}{c_{u}}
$$

We introduce Equation (29) into Equation (28), and obtain for the total deformation:

$$
u_{t}=u+\frac{u}{c_{u}} \cdot V
$$

For the total final specific deformation/strain $\varepsilon$, from Equation (30), we obtain:

$$
\varepsilon_{t}=\frac{u_{t}}{r_{0}}=\frac{u+\frac{u}{c_{u}} V}{r_{0}}=\frac{u}{r_{0}}+\frac{u}{c_{u}} \cdot \frac{V}{r_{0}}=\varepsilon_{0}+\varepsilon_{0} \cdot \frac{V}{c_{u}}
$$

The variation in the specific deformation, $\Delta \mathcal{E}$, from Equation (31) is then:

$$
\Delta \varepsilon=\varepsilon_{t}-\varepsilon_{0}=\varepsilon_{0} \cdot \frac{V}{c_{u}}
$$

In the case of an SMP2 moving with velocity $V$ in a direction different from $\mathrm{O}_{1}-\mathrm{O}_{2}$ (Figure 9), the influence on $\Delta \varepsilon$ will arise only from the component of $V$ in the direction $\mathrm{O}_{x}$, meaning that this will be smaller than in the case in Equation (32a). So the dynamic effect $+\Delta F_{L 12}$ created by $\Delta \mathcal{E}$ will modify the basic force $+F_{L 12}$ by a second factor $V / c_{u l}$ as follows:

$$
+\Delta F_{L 12}=+F_{L 12} \cdot \frac{V}{C_{u}}
$$


Since the force $F_{L 12}$ is modified as shown in Equation (32b), the force $F_{\mathrm{CC}}$ will also be modified, and hence the intensity of the quasielectric field $Q E$ will be modified by $\triangle Q E$.

This modification will normally be negligible, due to the negligible value of $V$ compared to the velocity of deformation $c_{u}$ of $S / M M$ from Equation (32a), based on the results from Sections 2 and 3.

It follows that the final size of $+\mathcal{E}$, and hence also the sizes of the resulting forces,$+-F_{L 12}$ and,$+-F_{L 21}$, cannot be significantly influenced, in the case of movement with normal velocity $V$ in the ETH, or between SMP2 and SMP1. However, for high speeds $V$, this influence $\Delta F_{L 12}$ cannot be neglected, as it results in important consequences at both the microscale and the macroscale. It affects the electric interaction forces $F_{\mathrm{CC}}$ and the gravitational forces $F_{\mathrm{DC}}$ according to [1] [2].

\subsection{FVTs of Mobile SMPs}

In this case, we consider the special situation of a mobile SMP with velocity $V$, which produces FVTs by means of rotation with speed $+\Omega$ around the axis Oz.

We take into consideration the fact consider that an immobile SMP will produce only simple, tangential circular deformations,$+-v$, but with an asymmetrical variation in the period $T$ associated with the FVs, as described in Sections 2 and 3.

In the case of a mobile SMP with velocity $V$ will result the superposition of two effects:

- basic tangential/circular deformations,$+-V$ as described in Section 3 for an immobile SMP;

- new tangential/longitudinal deformations $+\Delta v$ for a mobile SMP, which will be analysed below.

In an analysis of $+\Delta v$, we need to consider two phenomena that arise in conjunction with it:

1) The linear displacement of SMP with velocity $V$.

2) The concomitant rotation with speed $+\Omega$ for an SMPP or speed $-\Omega$ for an SMPN.

These two important phenomena are analysed below.

\section{1) Linear displacement of SMPs with velocity $V$ :}

The new deformation $+\Delta V$ appears as an result of the mobile/dynamic state of SMP2 moving with velocity $V$ in the direction of the axis $\mathrm{O} y$, normal to the direction $\mathrm{O}_{1}-\mathrm{O}_{2}$, relative to SMP1, which is considered to be immobile.

The velocity $V$ must be considered against the ETH, and in this case, the velocities $V$ of both parts of the analysed interaction need to be considered (Figure 9). This is because SMP1 will show the same effect if it moves with speed $V_{1}$.

First, we analyse the dynamic component $+\Delta v$ representing the change in the velocity $V$ of the surface $S / M M$ of SMP2 in the direction Oy, over a semiperiod $T / 2$, during which the normal/radial deformation,$+-u$ of the surface $S / M M$ of 
SMP2 develops, as described in Section 3. During T/2 time, SMP2 travels a distance $+\Delta y=+\Delta v$.

The deformation $+\Delta v$ in the area of mobile SMP2, will be transmitted further, not spherically as FVLs but cylindrically/radially as special FVTs, throughout the rest of the ETH. The deformation $+\Delta v$ will also reach SMP1, but with values $+\Delta \vec{v}$ that decrease as $1 / r^{2}$, according to Coulomb's law (as explained for,$+-u$ in Section 3).

The deformations $+\Delta v$ will develop longitudinally on the surface of a cylinder $\mathrm{CL}$, with its axis oriented in the direction of $\mathrm{O} y$, where CL is circumscribed to the sphere of SMP2 (Figure 9). The deformations $+\Delta v$ will be radially transmitted from this cylinder throughout the ETH, as described above.

We also note that the deformation $+\Delta v$ has a discrete, oscillating asymmetrical character in an period $T$, due to the vibrating nature of,$+-u$ with which it is associated, and this oscillating character of $+\Delta v$ will be transmitted to $\Delta \gamma$ and to associated $+\Delta F_{T 12}$.

The new effect $+\Delta v$ will created a change $+\Delta \gamma$ in the strains,$+-\gamma$ in ETH, including within the area AI of SMP1, and hence will produce a modification $+\Delta F_{T 21}$ in the force,$+-F_{T 12}$ acting on SMP1 (on a single EC), as discussed in Section 4 (Figure 9).

However, the specific deformations $+\Delta \gamma$ in the ETH resulting from the displacement of SMP2 with velocity $V$ will be simple as sense, but will oscillate in the direction of the velocity, $+V$. A change in the strain $+\Delta \gamma$ will be created by $+\Delta V$, produced by the mobile SMP2, and will be accompanied by the efforts variation $+\Delta \tau$, and by the resulting variation $+\Delta F_{T 21}$ of the forces,$+-F_{T 12}$.

For simplicity, we will refer to the modification $+\Delta \gamma$ that is due exclusively to the speed $V$, which we will denote simply as $+\gamma$.

In fact, by the deformations $+\Delta v$ and $+\gamma$, respectively, created by mobile SMP2, whose $S / M M$ will create a new dredging/training force $+F_{S T 21}$ on the ETH, as discussed in Sections 4 and 6.1. According to Newton's third law of the reciprocity of forces, and as explained in Section 4 , a similar change $+\Delta F_{T 21}$ in the force on SMP2 due to ETH will be created, where $+\Delta F_{T 21}=+\Delta F_{T 12}$. This force, $+\Delta F_{T 21}$, is the reaction force of the ETH on the mobile particle SMP2, in a direction opposed to its movement with velocity $V$.

The forces $+F_{T 21}$ vary in size with distance $r$ according to Coulomb's law, within the AI of the ETH, close to SMP1 (Figure 9).

In the case where SMP2 is in a dynamic/mobile state, travelling through the ETH with velocity $V$ directed tangentially to the SMP1 direction $\mathrm{O}_{1}-\mathrm{O}_{2}$, the above dredging/training effect of the ETH will appear. This new effect consists of a component $\Delta y=+\Delta v$, oriented orthogonally to the end of the representation segment of the deformation $u$, but acting in the $y$ direction, forming an angle $+\Delta \gamma$ (denoted as $\gamma$ ) with $u$ or with $z$ (Figure 9). $\Delta y$ represents the displacement along $y$ of the mobile particle SMP2 over the period $\Delta t=T / 2$, which corresponds to the expansion of $S / M M$ of SMP2, with $2 u / 2=u$. We can therefore write: 


$$
\Delta v=\Delta t \cdot V
$$

As a result, the $90^{\circ}$ angle between the deformation $u$ and the axis $\mathrm{O} y$ will become $90^{\circ}+\gamma$ (Figure 9), where $+\gamma$ represents the angle of deformation of the rectangular crystal lattice of the ETH. We can then write, using Equation (33):

$$
\gamma=\frac{\Delta v}{u}=\Delta t \cdot \frac{V}{u}
$$

The time $\Delta t$ during which the deformation $u$ is produced, with a velocity $c_{\mathrm{u}}$ of deformation of the $S / M M$ surface of SMP2, will be:

$$
\Delta t=\frac{u}{c_{u}}
$$

We introduce Equation (35a) into Equation (34) and obtain:

$$
\gamma=\Delta t \cdot \frac{V}{\Delta t \cdot c_{u}}=\frac{V}{c_{u}}
$$

Thus, the angle $+\gamma$ of deformation of the crystalline network of the ETH (Figure 9) is proportional to the velocity $V$ and inversely proportional to $c_{u}$. The velocity $c_{u}$ can be considered to be a constant, and this will be evaluated in the future.

Hence, the new effect of $V$, resulting in a strain $+\gamma$, will lead to a modification of the basic force,$+-F_{T 21}$, as described in Section 3, by a new force $+\Delta F_{T 21}$ with dredging effect between SMP2 and ETH, corresponding to the speed $V$ of SMP2 with respect to ETH. Assuming an elastic ETH, we can write:

$$
+\Delta F_{T 21}=K_{E T} \cdot \gamma
$$

where $K_{E T}$ is an elastic constant to be determined in future works.

Here, we assume that SMP2 has constant velocity $V$ over a long continuous period $t_{c o n}$, so that SMP2 in this movement can be considered a DC current.

The additional tangential force $+\Delta F_{T 12}=+\Delta F_{T 21}$ acting on SMP1, or on any other SMP in the ETH, will constitute the longitudinal component $H_{L}$ of the field $H$ which is generally referred to as magnetic but which in HM16 model we call the quasimagnetic field $Q H$. The component $Q H_{L}$ is caused by the movement of the SMP with longitudinal velocity $V$ via FVLs.

The presence of $Q H$ in an area $\mathrm{AI}$ can be associated with the presence in the AI of the specific strains $+\gamma$ of the ETH network (Figure 9).

\section{2) Rotation of SMPs at speed $+/-\Omega$ :}

This phenomenon of rotation of an SMPP/SMPN must be given the same importance as the velocity $V$ in point 1 ) to enable a correct understanding of the phenomenon of interaction between two SMPs.

We mention that for this mechanism of rotation with $+/-\Omega$ on the SMP scale, we do not have detailed information, but only the general knowledge of current physics, a multitude of phenomena involved in practically all chapters of physics.

The following paragraphs complements the discussion of HFVI in Section 2, and aims to ensure the compatibility of $+/-\Omega$ rotation phenomenon, with the 
rest of the interaction mechanisms of SMPs in HFVI, previously set out in [1] and in this paper as HFVI hypothesys. HFVI provides a logical explanation of the interaction process between the SMPs in the microcosm based on the mechanism of percussion forces, which form the basis of all phenomena in physics and nature.

For this purpose, we assume that the SMPP and SMPN are mainly characterised by their direction of rotation around the $\mathrm{Oz}$ axis with speeds $+\Omega$ and $-\Omega$, respectively.

We also assume that the two SMPs analysed previously, i.e. SMP1 and SMP2, have parallel $\mathrm{Oz}$ axes (Figure 9). Regarding the senses of the two $\mathrm{Oz}$ axes, we can admit the existence of both orientations of the analysed pair, but we will have to admit that the results of the interactions, as percussion forces, will differ in the two cases, but similar as a mode of interaction.

Based on these general considerations, we admit that the deformations +,- $v$ in the ETH produced by the two SMPs will arise from the surface $S / M M$ according to the mechanism described in Section 2.3.1., 2.6.

The deformations,$+-V_{s}$ is considered during the rotation,$+-\Omega$ of an SMP, and will form a tangent to an intermediate circle parallel to the equatorial plane of the SMP, with radius $r_{0}$. We therefore consider that,$+-V_{s}$ has an average value corresponding to a circle of radius $r_{0}$ at $45^{\circ}$ (Figure 9). These deformations,$+-V_{s}$ of $S / M M$ will also be transmitted to ETH in the form of deformations,$+-V$, which form the fundamental vibrations FVPP and FVPN, respectively.

For a SMPP, we assume that the deformation $V_{s}$ at time $t_{0}$ constitutes an initial deformation $+v_{s}$, that is clockwise and positive in the active phase, PHA. This will be followed by a counterclockwise deformation, $-V_{s}$, in the reactive phase, PHR, followed by another deformation $+v_{s}$, and so on, following the mechanism presented in Sec. 2.3.1., 2.6.

\section{3) Superposition of phenomena from 1) and 2) for SMPs:}

According to the mechanism proposed in Section 2.3.1., 2.6, deformations ,$+-V_{s}$ in the surface $S / M M$ and their counterparts,$+-V$ in ETH develop in two phases, i.e. an active and a reactive phase, without impeding the continuous rotations,$+-\Omega$ of the SMP. Only the active deformation $v$ will be transmitted by SMP to the ETH, giving a series of deformations $+v,+v,+v, \ldots$ acting on the ETH.

All of these deformations have frequency $f_{T} / \Omega$, or period $T$, and the deformations $+V_{s}$ correspond to the clockwise rotation of the radius $r_{0}$ of the SMP around the axis $\mathrm{Oz}$. These properties will be transmitted to the ETH as deformations,$+-v$ of the ETH with $v_{s}=v$ (Figure 9).

In the case of an SMPN, the phenomenon is similar to that for an SMPP, but with a reversal of the rotation sense, $-\Omega$.

Hence, the rotation of the SMP will give rise to a series of deformations $+v,+v$, $+V, \ldots$ with frequency $f_{T} /+\Omega$, as in point 2 ).

As a result, a two deformations vector summation will occur: one of the new 
deformations $+\Delta V$ from $V$ in period $T$ from point 1 ), and ones on the base from rotations from 2$)(+v)$. Thus, a series of cumulated deformations $+\Delta v_{c}$ will result, referred to here, as cumulative deformations:

$$
\Delta v_{c}=v+\Delta v
$$

Or their associate $\gamma$ strains:

$$
\Delta \gamma_{c}=\gamma_{R}+\Delta \gamma_{V}
$$

These cumulative deformations $+\Delta v_{c}$ will have as their starting points the surface of a cylinder CL resulting from the translation of the largest circle of the sphere of SMP2, which is moving with velocity $V$ along the Oy axis.

In this way, the trajectories of the deformations $+\Delta v_{c}$ will appear as spirals that evolve in time after their departure from the surface of the cylinder CL. These deformations will be transmitted to the overall ETH (Figure 9).

The sense of rotation of the spirals will depend on whether the particle is an SMPP or an SMPN. The mechanical effect of these deformations $+\Delta v_{c}$ or of associated strains $+\gamma$ will be the percussion forces $+\Delta \boldsymbol{F}_{C 12}$ acting on SMP1. These are also cumulative vectorial forces, $F_{C 12}$, as are vectorial also deformations $\gamma$ of their origin (36c):

$$
+\Delta \boldsymbol{F}_{C 12}=+,-\boldsymbol{F}_{R 12}+\Delta \boldsymbol{F}_{V 12}
$$

The force $+\Delta \boldsymbol{F}_{C 12}$ will form a field in ETH called the $H$ field, which in mainstream physics is referred to as magnetic but which in the HM16 model we call the quasimagnetic field $Q H$. As can be seen from Equation (36c), the field has two components: rotational $Q H_{R}$ and velocity $Q H_{V}$ components:

$$
Q \boldsymbol{H}=Q \boldsymbol{H}_{R}+Q \boldsymbol{H}_{V}
$$

The presence of $Q H$ in an area $\mathrm{AI}$ can be associated with the presence in the AI of the specific cumulative strains $+\gamma_{c}$ from (36c) of the ETH network (Figure 9).

The force $+\Delta F_{C 12}$ will have the same effect as the Lorenz force $F_{L}$, of transverse thrust/dredging of SMP2, created by a field $H$ on an electric charge,$+-q$ that is in transverse motion with velocity $V$ (Figure 9 ).

The force $+\Delta F_{C 12}$ is tangential and spiral (based on the two components shown in Equation (36d)), and will also produce a cumulative rotational moment $+\Delta M_{C 12}$, which acts on SMP1 as explained in Section 4 . This moment $+\Delta M_{C 12}$ will contain the dredging effect of the force $+\Delta F_{V 12}$, thus creating an additional quantum effect on SMP1.

Note that these forces will act simultaneously on SMP1, i.e. both the dredging force $+\Delta F_{V 12}$ arising from the velocity difference $V$ between the two components, and the forces,$+-F_{R 12}$ due to the rotations from FVTs produced by moving SMP2, as shown in Equation (36d) and described in Section 4.

In the case of a group GSMP2, the individual force $+\Delta F_{C 12}$ will have a considerable size, since it is proportional to the number $n$ of SMPs in GSMP2.

Here, we consider the contribution to the forces $+\Delta F_{C 12}$ from the central string 
$\mathrm{ST}_{0}$ in the direction $\mathrm{O}_{1}-\mathrm{O}_{2}$ of the ECs, as discussed in Section 2 (Figure 9). The total resultant will be a tangential force $F_{\mathrm{TCC}}$ which is calculated by considering the set of paths $l_{i}$ as set out in [8].

The orientation of the $Q H$ field will depend on the direction and sense of the velocity $V$ of the SMP2, and also on the sense of rotation $+/-\Omega$ of SMP2, as follows: it will create cumulative, oscillating strains,$+-\gamma_{c}$ in ETH, inclined in the direction and sense of the velocity $V$, containing also rotating strains $+\gamma_{R}$ in the sense of $+/-\Omega$ in the AI area of SMP1 (Figure 9). The cumulative effect will result in a complex $+\Delta \gamma_{c}$ in the overall ETH.

At the same time, the $Q H$ field represents the classical properties of the magnetic field $+H$, for the inclination/orientation angles $+\gamma_{\mathcal{c}}$, and rotation,$+-\Omega$ in the Oxyz reference frame, according to Fleming's rule of magnetic induction, and depending on the type of particle (SMPP or SMPN). Thus, the oscillating force $+\Delta F_{C 21}$ will be of the Lorenz type, and will be exerted on SMP1 due to the velocity $V$ and rotation $+/-\Omega$ of SMP2, and due to the effect of the oscillating moment $+\Delta M_{C 21}$. The force $+\Delta F_{C 21}$ and the moment $+\Delta M_{C 21}$ will be imprinted to SMP1, over its own rotation vibrations,$+-u / v$ and rotations $+/-\Omega$, resulting a complex effect containing forces/percussions $F / p_{p}$ associated to displacements $u / v$, all of a quantum nature, because forces/percussions/displacements, act on a single EC.

But the dredging effect $+\Delta F_{\mathrm{CT} 21}$ of mobile SMP2 will be transmitted to SMP1 upon his $\gamma_{\mathrm{c}}$, with a decreased effect, with $r$ as a Coulomb type effect (Figure 9).

If we assume that SMP2 has a constant velocity $V$ over a long period $t_{c o n}$, it can be considered a linear DC current, given by a string of SMP2 of P or N type, having the same orientation of $\mathrm{Oz}$ axes, but only considering the concomitant phenomenon of the rotation of SMP2 with speed,$+-\Omega$ (Figure 9).

In the case of a moving SMP2 with velocity $V$ describing a circle as part of a spiral, with a constant radius $R$ over a long period $t_{c o n}$, the SMP2 can be considered a solenoid containing a DC current, with a specific strong $Q H$ field at the inner part of the radius $R$ (Figure 9). This strong $Q H$ field is caused by the concentration within a limited zone of radius $R$ of all the paths of the deformations $u / v$, from the half of the linear current case, but only considering the phenomenon of rotation of SMP2 with speed,$+-\Omega$. This results in a complex effect upon SMP1, involving forces $F$ and displacements $u / v$ of a quantum nature.

\subsection{Energetic Aspects}

As set out in Sections 6.1 and 6.2, the energy $\Delta E_{U}$ is related to the percussions ,$+-p_{n}$ and,$+-p_{t}$ including the related forces,$+-F_{L 21}$ and,$+-F_{T 21}$, respectively, which change their point of application and produce mechanical work $L$, which will come from the potential energy $U$ of the SMP2-GSMP1 system, energy left since its creation. Such energy will be released by the movement of SMP2 at speed $V$ (Figure 9). This situation also corresponds also to the proper application of the general law of Lorenz force [10] [11]: 


$$
F=q E+q v \times B
$$

The potential $U$ energy of the SMP2-GSMP system is stored in the $Q E+Q H$ fields.

In this case, the energy $+\Delta E$ taken from the potential energy $U$ is associated with the SMP2-GSMP1 system of MPs disposed in the neighbouring AI of SMP2, from ETH. The transfer will take place through the $Q E$ or $Q H$ fields. These quasielectric and quasimagnetic fields create the percussion forces,$+-p_{n}$ and,$+-p_{t}$ and the associated forces,$+-F_{L 21}$ and,$+-F_{T 21}$, to give the associated moments $+M_{L 21}$ and $+M_{T 21}$, fields, which will imprint to SMP2 the speed $V$, or the speed increase $+\Delta V$ and the rotation increase $\Delta \Omega$ respectively (Figure 9).

It should be noted that the specific deformations,$+-\varepsilon$ and,$+-\gamma$ corresponding to deformations,$+-u$ and,$+-V$ may vary in space. The deformations,$+-u$ and ,$+-V$ appear in the ETH due to the change in the reference distance $r$, through $V$ between two SMPs, which are initially immobile in the ETH (also considered immobile). These deformations will be produced by one of the SMPs, which moves at velocity $V$ through the ETH.

The initiation of the displacement with velocity $V$ through the ETH of SMP2, occurs under the influence of the field $Q E+Q H$. SMP2 also gains rotational movement with a change of speed $\Delta \Omega$, as a subtle quantum effect (Figure 9).

\section{Mechanism of PH Emission and Movement with Speed $c$ in the ETH}

Here, we discuss a possible approach to compiling and operating a $\mathrm{PH}$ that travels freely into space, and therefore within the ETH, at speed $c$.

We assume that the $\mathrm{PH}$ can be considered an ESMP, i.e. that the basic composition consists of ECs of PCs (including quarks). Simple etherons (ETs) inside any SMP are likely to be grouped into PCs. We assumed in [1] that the composition of ECs and their grouping within ETH is based on a crystal type body, according to the HM16 ether model, Type A. An EC cell comprises a small number (maybe two to eight) of ETs, while a PC comprises a larger number of ETs.

We assume that the $\mathrm{PH}$ comprises a large number of etheronic cells PCs of particle type, that are assembled in the form of a $\mathrm{PH}$ compact body, constituting an autonomous PAC as described in Section 1 (Figure 10).

In HFVI, we admit that $\mathrm{PH}$ moves through the ETH at a specific speed $c$, without training in its displacement, the ECs cells encountered, which remain in place as ECs at the same fixed point $M$, like initial ECs, at the same $M$ point, after PH's passage through point $M$. The $\mathrm{PH}$ behaves like a vortex that entrains ECs only in its vortex movement only within its own volume $V$, along its path.

This mode of movement of a PH in the ETH without transporting ECs has also been considered in regard to the MH16 model [1], a hypothesis that became useful only here for the best functioning of the $\mathrm{PH}$.

We admit in HFVI that the PH can be created by a pair of particles SMP1/SMP2 or a GMP that is organised in a certain stable form for a while. 


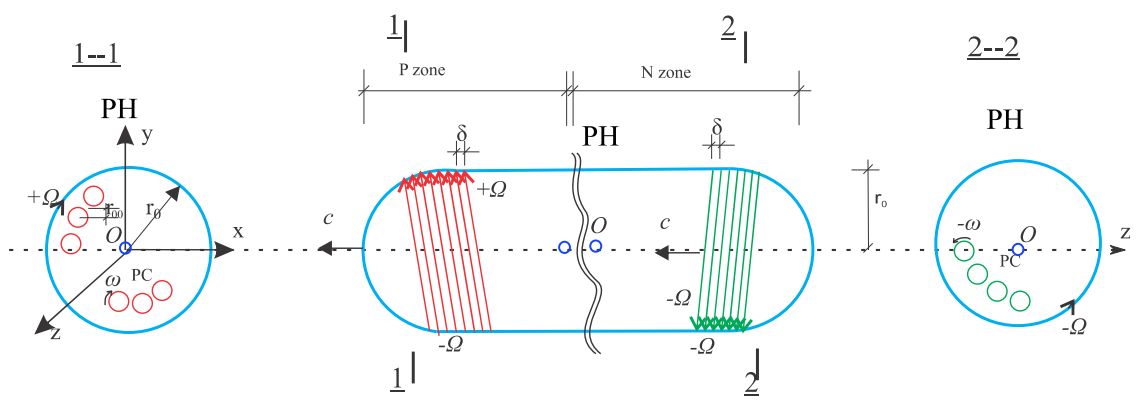

a). Photon PH model, Variant $\mathrm{A}$ as a single particle

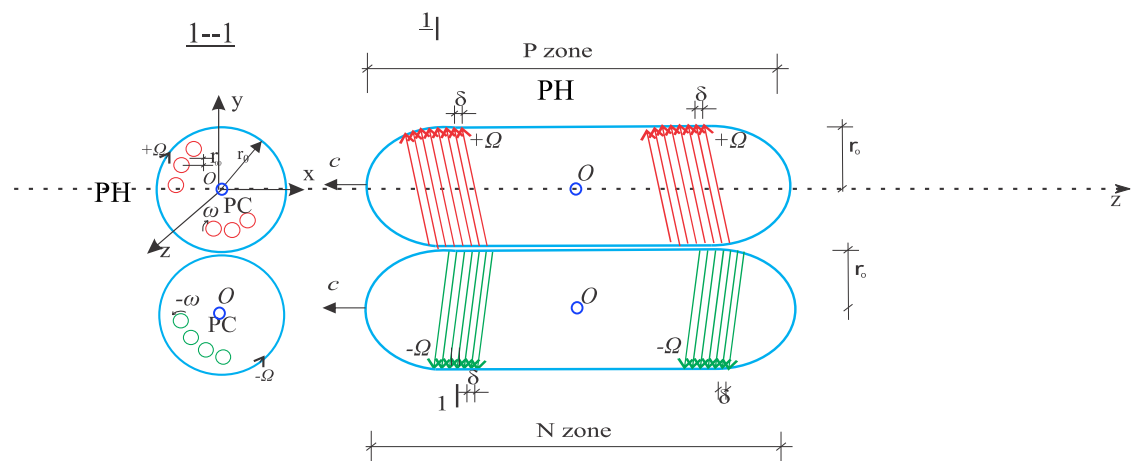

b). Photon PH model, Variant B as a double particle

Figure 10. Physical mechanism of movement of a $\mathrm{PH}$ in the ETH with velocity $\mathrm{c}$ based on the hypothesis of scrubbing.

A PH is created when the energy level $E$ of an MP or GMP decreases by $-\Delta E$ for some reason, usually after a prior energy increase of $+\Delta E$. The creation of a $\mathrm{PH}$ consists of the expulsion at the speed of light $c$ of a PH by a pair of MPs, and this $\mathrm{PH}$ will take the equivalent energy $E_{p h}$ from the MPs or GMP, where $E_{p h}=$ $\Delta E$. In the HM16 ether model, we assume that the received energy $\Delta E$ is stored in the $\mathrm{PH}$ within the PP, permanently in a vibrational state with vortices of rotation speed $\omega$, either individually or grouped into certain PC/quark-type.

In the case of the creation of a PH with energy $E_{p h}$, the mass $m$ of the MPs will also have to be reduced by $\Delta m=E_{p h} / c^{2}$, corresponding to a certain number of PC cells. This is Variant 1 for the transformation of the mass $m$ of some PCs into the energy transmitted to $\mathrm{PH}$, since the PCs are also the carriers of the mass $\Delta m$ of the MP/SMP.

The energy difference $-\Delta E$ will be entirely found in the $\mathrm{PH}$ photon, as vortices/vibrations of ECs/PCs, temporarily contained in the PH's volume that moves at speed $c$ and, likewise, the mass difference $\Delta m$ must also be found in $\mathrm{PH}$.

Since at least one SMPP and one SMPN are involved when the PH is created by annihilating pairs of particles, the resulting $\mathrm{PH}$ will have to comprise two areas with characteristics specific to these SMPs. Additional support for this mechanism also arises from the phenomenon of the creation of pairs (Section 2.5), when a PH turns into an SMPP and an SMPN.

We admit in HFVI that the PH will have an ellipsoid shape, including a positive zone (PZ) in which its own sense of rotation will be clockwise (considered 
positive), and a negative zone (NZ), in which the sense of rotation will be counter clockwise (Figure 10).

These two areas can be coupled either in series or in parallel (Figure 10(a) and Figure 10(b)).

In photon Variant VPH 1, we assume that the two zones ( $\mathrm{PZ}$ and $\mathrm{NZ}$ ) are coupled in series, forming a single ellipsoid (Figure 10(a)), while in Variant VPH 2, we assume that they are coupled in parallel to form a double ellipsoid (Figure $10(\mathrm{~b}))$.

In photon Variant VPH 3, we assume that the two zones ( $\mathrm{PZ}$ and NZ) are complete separated, forming two simple ellipsoids (Figure 10(a)-adapted), which are moving separately, which correspond to simultaneous emission of two PHs.

The energy $\Delta E$ will be transmitted over a short period of time to the $\mathrm{PH}$ by two types of kinetic: kinetic energy of displacement $E_{d i s}$ at speed $V$, and rotation energy $E_{\text {rot }}$ at rotation speed $\Omega$.

These two movements, one involving linear advance, and the other involving the rotation of the $\mathrm{PH}$ in the ETH, will be produced by periodic percussion forces $p_{s n}$-normal and $p_{s t}$-tangential applied to the $\mathrm{PH}$ by the $S / M M$ of the two MPs. Percussions will be applied via associated vibrations,$+-u$ and,$+-v$ of the $\mathrm{PCS} / M M$ in final contact with $\mathrm{PH}$ at the time of its detachment from the two MPs (Figure 10).

After the detachment of the PH from the SMPs, it will produce in ETH its own percussions $p_{s}$ in the same way as an ESMP, which will simultaneously create own FVLs and FVTs of the ECs of the surrounding ETH.

The movement of the $\mathrm{PH}$ at speed $c$ starts with the two SMPs imprinting of the $\tau$-type efforts on its lateral surface $S / M M$, creating a sudden rotation of the $\mathrm{PH}$ with the speed,$+-\Omega(v)$, which will be applied differently to the two zones, $\mathrm{PZ}$ and $\mathrm{NZ}$ of the $\mathrm{PH}$ (Figure 10).

This correlation will also be guided by the nuclei NUs, considering the role of those NUs of the SMPs, involved in the creation of the $\mathrm{PH}$, according to the SMPAC hypothesis presented in Section 1. Thus, the speed $c$ resulting from the advancement process by its double screwing in ether will be imprinted to $\mathrm{PH}$ (Figure 10).

Simultaneously, the efforts of type $\sigma$ from the percussions $p$ acting on the back surface $S / M M$ of the $\mathrm{PH}$ may contribute to the launch/creation of the $\mathrm{PH}$ by imprinting speed $c$.

We put forward here the hypothesis of scrubbing (HS) as a valid and feasible in the physical reality in which the PH starts and moves through the ETH due to a screwing process in the ETH. We assume here that the $\mathrm{PH}$ has a hydrodynamic ellipsoid shape, and to simplify the calculations we will approximate this ellipsoid by an elongated cylinder of equivalent volume (Figure 10).

This process of displacement at speed $c$ occurs due to the rotation of this ellipsoid /cylinder around its longitudinal axis with rotation speed/frequency $\Omega / f$. We assume that the surface/membrane $S / M M$ of the PH's ellipsoid, has certain regular striations or protrusions, which may play the role of a spiral helical thread 
arranged around and along the ellipsoid, differentiated by the directions of rotation in the two zones ( $\mathrm{PZ}$ and $\mathrm{NZ}$ ) of the $\mathrm{PH}$, with step size $\delta$ specific to each $\mathrm{PH}$ (Figure 10).

The HS is based on a simple process that facilitates the advance of a certain body into a compact, penetrating environment, by scrubbing. The validity of the HS can be initially confirmed by the theoretical results it provides, as shown below.

In the HS, for a $\mathrm{PH}$ with frequency $v$ (here we use the Greek $v$ rather than $f$ ) and a spiral step $\delta$, moving through the ETH at speed $c$, we have the equation:

$$
c=\delta v
$$

We will denote the initial mass of the $\mathrm{PH}$ as $m_{P}$ and apply to it the equation of total energy $E_{p h}$ :

$$
E_{P}=m_{p h} c^{2}
$$

If we also take into account Planck's law [11], we can write:

$$
h v=E_{p h}
$$

Combining (39) with (40) gives:

$$
\begin{gathered}
h v=m_{p h} c^{2} \\
m_{p h}=\frac{h v}{c^{2}}
\end{gathered}
$$

If we multiply by $c$ in Equation (41a) we get:

$$
m_{p h} c=\frac{h v}{c}=\frac{h}{\lambda}
$$

We introduce $c$ from Equation (38) into Equation (41b) to give:

$$
m_{p h} \delta v=\frac{h v}{c}
$$

Simplification with $v$ in Equation (32a) gives:

$$
m_{p h} \delta=\frac{h}{c}
$$

From Equation (44), given that the product $c . m_{p h}$ represents the pulse $p_{p h}$ of the $\mathrm{PH}$, we obtain:

$$
\delta=\frac{h}{m_{p h} c}=\frac{h}{p_{p h}}
$$

Equation (45) can be generalised using de Broglie's theory to any MP of mass $m$, pulse $p$ and speed $v<c$ moving through the ether, also through the phenomenon of scrubbing with its own step $\delta$, giving:

$$
\delta=\frac{h}{m v}=\frac{h}{p}
$$

We now apply Equation (45) to the case of a photon with any frequency $v$, for which the resting mass is $m_{p h}$ in Equation (41b): 


$$
\delta=\frac{h}{\frac{h v}{c^{2}} c}=\frac{c}{v}=\lambda
$$

So for a PH of frequency $v$ moving through the ether by scrubbing, the thread step $\delta$ is equal to the wavelength $\lambda$, equivalent to the frequency $v$. This result confirms the well-known physical phenomenon of advancing a drill by rotation into a compact environment, and hence the HS is theoretically confirmed.

\section{Conclusions and Consequences}

In this article, we have presented a new way of constructing SMPs as complex cells such as LCs, with their own MM, PP, and NC.

A complex hypothesis called HFVI for the interaction between SMPs was developed based on periodic percussion forces,$+-p_{p}$ created by the FVs produced by any SMP in the ETH upon a single EC. We have shown that these percussions ,$+-p_{p}$ are the smallest forces occurring in the interactions between any two SMPs, and are periodic with associated deformations,$+-u /+,-v$, at a frequency $f$, with energy of,$+-p_{p}$ upon a single EC, representing Planck's constant $h$ as a physical phenomenon related to quantum actions between particles.

Based on the FVs of SMPs, which create periodic linear,$+-u$ and transversal ,$+-v$ deformations in the ETH, and which vibrate and act simultaneously, it was possible to establish the physical nature of the electrical charges,$+-q$, given by the specific rotation $+/-\Omega$ and vibration of an SMPP and of a SMPN, i.e. positively and negatively charged SMPs, respectively, starting from the moment of creation of this the pair, due to a $\mathrm{PH}$ photon.

Based on this knowledge of the physical nature of the electrical charges,$+-q$ generated by SMPs producing specific FVs, it was possible to confirm the manner of interaction between two SMPs through,$+-F_{L 21}$ forces as completed Coulomb's type attraction/repulsion forces $F_{\mathrm{CC}}$ between two SMPs, via normal,$+-p_{n}$ percussion forces upon a single EC generated by specific longitudinal FVL vibrations.

The nature of magnetic interaction could also be confirmed in the case of movements with velocity $V$ between two SMPs, via tangential percussion forces ,$+-p_{t}$, upon a single EC generated by specific FVTs created by the particles.

So a mechanism for interactions between SMPs was proposed based on the velocity $V$ and the rotation speed $\Omega$ of the SMPs. This results in explaining the properties of the quasielectric field $Q E$, given by specific ordered/oriented strains $+\mathcal{E}$, and the properties of the quasimagnetic field $Q M$, given by specific ordered/oriented strains $+\gamma$ created by a GSMP of the same type of particle $(+/ \mathrm{P}$ or $-/ \mathrm{N})$ that are ordered and moving together. This mechanism also considers the associated forces $+F_{L 21}$ and $+F_{T 21}$, of the,$+-p_{n}$ and,$+-p_{t}$ percussions, for an SMP in the ETH.

Based on the periodic percussions,$+-p_{p}$, a scrubbing mechanism was postulated for the start and movement at speed $c$ in the ETH of a photon $\mathrm{PH}$, which 
can be considered as being made up of two zones with opposite rotations traveling without transporting matter, only energy.

Based on these new results, the validity of our complex hypothesis HFVI is theoretically confirmed.

As a general consequence, it follows that there is a need for the HM19 model of the ether and the above HFVI hypothesis to be accepted into mainstream physics. Concomitantly it must renounce at classical SRT, GR, gravity, electromagnetism theories.

Therefore will resulting in a new NPHM19 physics, based on the ether ETH, starting from the HM16 model, which will be able to provide a physical explanation of quantum mechanics as true classical mechanics phenomena in microcosm based on periodic percussion forces $p_{p}$ upon a single EC NPHM19 can be developed on these new basis by a broad community of open-minded physicists released by SRT, who will be necessary to guide the main theoretical concerns and experimental research on NPHM19 towards new areas.

It will obviously be necessary to correct certain assumptions, reasoning or calculations in our previous works, that may prove to be incorrect in future. In this way, NPHM19 will be able to give correct new answers to some of the many unresolved questions and problems in physics, astronomy, chemistry, biology and other fields. However, some unanswered questions will still remain and new questions will also constantly arise.

We believe that the human mind is able to encompass, understand and resolve situations in nature at increasingly profound levels, both at the microcosmic and macrocosmic levels, but only in a step-by-step way; a definitive ultimate truth is probably too far away to be touched.

\section{Acknowledgements}

The first author gratefully acknowledges initial advice on the subject and encouragement from his late professor N. Barbulescu, a Sommerfeld follower. He is also sincerely grateful to late Prof. P. Mazilu from TUCB Bucharest, for his rigorous lessons on rationality, and is indebted to Gen. Prof. G. Barsan, Col. Prof. Al. Babos from LFA Sibiu, Prof. D. Stoicescu from ULBS Sibiu, Prof. D. Siposan from MTA Bucharest, and Ms Veronica Has from Bucharest, for their support.

\section{Conflicts of Interest}

The authors declare no conflicts of interest regarding the publication of this paper.

\section{References}

[1] Has, I. and Miclaus, S. (2017) Physics Essays, 30, 45-56. https://doi.org/10.4006/0836-1398-30.1.45

[2] Has, I., Miclaus, S. and Has, A. (2017) Physics Essays, 21, 303-312. https://doi.org/10.4006/1.3038751

[3] Has, I., Miclaus, S. and Has, A. (2015) American Journal of Modern Physics, 4, 
97-108. https://doi.org/10.11648/j.ajmp.20150403.11

[4] Has, I., Miclaus, S. and Has, A. (2014) Optics, 3, 24-32. https://doi.org/10.11648/j.optics.20140304.11

[5] Has, I., Miclaus, S. and Has A. (2018) Journal of Applied Mathematics and Physics, 6, 1886-1895. https://doi.org/10.4236/jamp.2018.69160

[6] Has, I., Miclaus, S. and Has, A. (2019) Journal of Physics. Conference Series, 1251, 1-24. https://doi.org/10.1088/1742-6596/1251/1/012020

[7] Has, I., Miclaus, S. and Has, A. (2018) Journal of Applied Mathematics and Physics, 6, 1507-1521. https://doi.org/10.4236/jamp.2018.67127

[8] Has, I., Miclaus, S. and Has, A. (2019) Journal of Modern Physics, 10, 1090-1124. https://doi.org/10.4236/jmp.2019.109071

[9] Constantinescu, L., Tanasescu, P., Radulescu, M., Doicin, D., Rosca, V., Enescu, D., Candea, I., Constantinescu, P. and Barbu, M. (1965) Geophysical Prospecting Vol. II. Editura Tehnica, Bucharest, 215-286. (In Romanian)

[10] Barbulescu, N. (1962) Elements of General Physics. Editura Didactica \& Pedagogica, Bucharest, 283-344. (In Romanian)

[11] Spolski, E.V. (1954) Atomic Physics I. Editura Tehnica, Bucharest, 234-240. (In Romanian) 